\title{
Complex organic molecules in organic-poor massive young stellar objects
}

\author{
Edith C. Fayolle ${ }^{1,2}$, Karin I. Öberg ${ }^{2}$, Robin T. Garrod ${ }^{3}$, Ewine F. van Dishoeck ${ }^{1}$, and Suzanne E. Bisschop ${ }^{4,5}$ \\ ${ }^{1}$ Leiden Observatory, Leiden University, PO Box 9513, 2300 RA Leiden, The Netherlands \\ e-mail: efayolle@cfa.harvard.edu \\ 2 Harvard-Smithsonian Center for Astrophysics, 60 Garden Street, Cambridge, MA 02138, USA \\ 3 Center for Radiophysics and Space Research, Cornell University, Ithaca, NY 14853-6801, USA \\ 4 The Centre for Star and Planet Formation, Natural History Museum of Denmark, University of Copenhagen, Øster Voldgade 5-7, \\ 1350 Copenhagen K., Denmark \\ 5 The Centre for Star and Planet Formation, Niels Bohr Institute, Juliane Mariesvej 30, 2100 Copenhagen Ø., Denmark
}

Received 22 November 2013 / Accepted 22 October 2014

\section{ABSTRACT}

\begin{abstract}
Context. Massive young stellar objects (MYSOs) with hot cores are classic sources of complex organic molecules. The origins of these molecules in such sources, as well as the small- and large-scale differentiation between nitrogen- and oxygen-bearing complex species, are poorly understood.

Aims. We aim to use complex molecule abundances toward a chemically less explored class of MYSOs with weak hot organic emission lines to constrain the impact of hot molecular cores and initial ice conditions on the chemical composition toward MYSOs. Methods. We use the IRAM $30 \mathrm{~m}$ and the Submillimeter Array to search for complex organic molecules over $8-16 \mathrm{GHz}$ in the $1 \mathrm{~mm}$ atmospheric window toward three MYSOs with known ice abundances, but without luminous molecular hot cores.

Results. Complex molecules are detected toward all three sources at comparable abundances with respect to $\mathrm{CH}_{3} \mathrm{OH}$ to classical hot core sources. The relative importance of $\mathrm{CH}_{3} \mathrm{CHO}, \mathrm{CH}_{3} \mathrm{CCH}, \mathrm{CH}_{3} \mathrm{OCH}_{3}, \mathrm{CH}_{3} \mathrm{CN}$, and $\mathrm{HNCO}$ differ between the organic-poor MYSOs and hot cores, however. Furthermore, the N-bearing molecules are generally concentrated toward the source centers, while most $\mathrm{O}$ - and $\mathrm{C}$-bearing molecules are present both in the center and in the colder envelope. Gas-phase $\mathrm{HNCO} / \mathrm{CH}_{3} \mathrm{OH}$ ratios are tentatively correlated with the ratios of $\mathrm{NH}_{3}$ ice over $\mathrm{CH}_{3} \mathrm{OH}$ ice in the same lines of sight, which is consistent with new gas-grain model predictions.

Conclusions. Hot cores are not required to form complex organic molecules, and source temperature and initial ice composition both seem to affect complex organic distributions toward MYSOs. To quantify the relative impact of temperature and initial conditions requires, however, a larger spatially resolved survey of MYSOs with ice detections.
\end{abstract}

Key words. ISM: abundances - ISM: molecules - astrochemistry

\section{Introduction}

Organic molecules containing more than six atoms, the so-called complex organics (Herbst \& van Dishoeck 2009), are commonly found in the warm and dense gas $\left(T>100 \mathrm{~K}, n>10^{6} \mathrm{~cm}^{-3}\right)$ around young stellar objects (YSOs), so-called molecular hot cores (e.g., Blake et al. 1987; Cazaux et al. 2003; Fuente et al. 2005). Abundances and abundance ratios of complex organics are found to vary substantially between (Helmich \& van Dishoeck 1997) and within YSOs (e.g., Wyrowski et al. 1999). This suggests that formation and destruction routes are highly environment specific and that there is a sensitive dependence of the complex organic chemistry on chemical and physical initial conditions. In addition, different filling factors of the warm gas should play a role if there the complex organic products of cold and hot chemistry differ there.

The potential environmental dependencies and chemical memories lead to complex organics having a great potential as probes of the current and past physical and chemical conditions where they are found (Nomura \& Millar 2004). Their potential utility is further increased by the fact that most complex organic molecules present large numbers of lines, spanning most excitation conditions found in space. Complex molecules are also of high interest for origins of life theories since they are the precursors of even more complex prebiotic material (Ehrenfreund \& Charnley 2000). Using molecules as probes of physical conditions and advancements in prebiotic evolution from organics both rely on a detailed understanding of complex organic chemistry. The formation and destruction mechanisms and rates of most complex organics are, however, poorly constrained.

The formation of organic molecules around massive YSOs (MYSOs) was first thought to proceed through gas phase reactions in dense hot cores, following evaporation of ice grain mantles (e.g., Charnley et al. 1992). Recent laboratory experiments and modeling efforts point now toward a more complicated sequential scenario that relies to a greater extent on surface formation routes on submicron-sized dust particles. Herbst \& van Dishoeck (2009) classify complex organic molecules in terms of generations according to the following scenario. In interstellar clouds and in the deeply embedded early phases of star formation, atoms and molecules accrete or form on the surface of dust grains, building up an icy mantle of simple species like $\mathrm{H}_{2} \mathrm{O}, \mathrm{CH}_{4}$, and $\mathrm{NH}_{3}$ (Tielens \& Hagen 1982). This icy mantle is processed at low temperature by atoms, which can diffuse even at the low temperatures in cloud cores, creating the zeroth generation of organic molecules. A good example of these species 
is $\mathrm{CH}_{3} \mathrm{OH}$, which is efficiently formed at low temperature by the hydrogenation of CO ice (Watanabe \& Kouchi 2002; Watanabe et al. 2003, 2004; Fuchs et al. 2009; Cuppen et al. 2009). Firstgeneration complex organics form when heating the cold envelope up by the increasing luminosity of a central YSO and is due to a combination of photoprocessing of the ice resulting in radical production and a warming up (20 to $100 \mathrm{~K})$ of the grains, thereby enhancing the mobility of radicals and molecules (Garrod et al. 2008; Öberg et al. 2010). When the icy grains move inward and reach a region warmer than $100 \mathrm{~K}$, the icy mantle evaporates, bringing the zeroth- and first-generation organics into the gas phase, where additional chemical reactions give rise to the formation of the second-generation complex organics (e.g., Charnley et al. 1992; Doty et al. 2002; Viti et al. 2004).

In the proposed scenario of complex molecule formation, the initial ice mantle plays a critical role. The exact composition of this ice may therefore have a strong effect both on the product composition of formed organics and on their overall formation efficiency. Garrod et al. (2008) and Öberg et al. (2009) find, for example, that $\mathrm{CH}_{3} \mathrm{OH}$ ice is a key starting point for most complex organic formation. Rodgers \& Charnley (2001) used a hot core chemistry model to show that the relative amount of $\mathrm{NH}_{3}$ in the ice has a large impact on the $\mathrm{CH}_{3} \mathrm{CN} / \mathrm{CH}_{3} \mathrm{OH}$ protostellar abundance ratio. Observationally testing these relationships would provide key constraints on the formation pathways of complex organic molecules.

Isolated MYSOs with warm inner envelopes are good laboratories for testing this hypothesis as these sources are bright enough to observe a wide variety of organics and some of them present ice features from the cold outer protostellar envelope (Gibb et al. 2004). Sources presenting both complex gas and ice features are, however, rare as the sources need to be evolved to possibly display a bright hot core chemistry accessible to current observational facilities and young enough such that the ice material has not been completely consumed by accretion, warm up, and envelope dispersal. In the massive YSO sample studied by Bisschop et al. (2007), only three hot cores present ice spectra (see Table 1). Such a small number prevents any analysis of the correlation between ice and gas content and justifies our search for other objects that display both ice features and gas phase organics.

To extend the sample of sources with both complex organics and ice observations, we look for gas phase organics species around non-hot core MYSOs (absence or low-level of hot $\mathrm{CH}_{3} \mathrm{OH}$ emission) that also have ice observations available from the literature. These sources are called from now on organic-poor MYSOs (poor in lines of organic molecules). Complex molecule observations in such objects may additionally shed light on the conditions under which different kinds of complex molecules can form, i.e. which molecules require the presence of a hot core to be abundant.

Massive objects NGC 7538 IRS9, W3 IRS5, and AFGL490 have been observed in the mid-infrared by the Infrared Space Observatory (ISO) and analyzed systematically for ice abundances by Gibb et al. (2004) and references therein. NGC 7538 IRS9 is a $6 \times 10^{4} L_{\odot}$ luminous object located in Perseus. It is close to hot core source NGC 7538 IRS1 and displays at least three bipolar outflows, evidence for accretion (Sandell et al. 2005), and a hot component close to the central object. W3 IRS5 is associated with five YSOs, two of which are massive (van der Tak et al. 2005; Megeath et al. 2005; Rodón et al. 2008; Chavarría et al. 2010). It has a luminosity of $17 \times 10^{4} L_{\odot}$ and presents strong S-bearing molecular lines
(Helmich et al. 1994). AFGL490 is a very young medium-mass YSO of $4.6 \times 10^{3} L_{\odot}$, in transition to a Herbig Be star, which drives a high-velocity outflow (Mitchell et al. 1995) and shows evidence of a rotating disk (Schreyer et al. 2006). Since these sources are considered to potentally be at an earlier evolutionary stage than typical hot-core sources, it is difficult to predict the chemical complexity and the spatial emission of the organics that could be observed in these sources.

In this study we use a combination of single-dish IRAM $30 \mathrm{~m}$ data and spatially resolved observations from the Submillimeter Array ${ }^{1}$ (SMA) to search for organic molecules around these three MYSOs and report on their complex organic abundances in the cool protostellar envelope and in a warmer region closer to the star. A subset of these data was used in the Öberg et al. (2013) to study the detailed radial distribution of molecules in NGC 7538 IRS9, while the present study focuses on the overall detection rate of organics in these organic-poor sources, and on how they compare with ice abundances and traditional hot core chemistry. The paper is organized as follows. The observations are described in Sect. 2, and the results of the line analysis are shown in Sects. 3.1-3.3. The chemistry in our sample is compared to the chemistry in traditional hot-core sources in Sect. 3.4. Section 3.5 presents correlation studies between ice and gas column densities and abundances, testing the impact of initial ice compositions on the complex chemistry. A discussion of the use of these line-poor sources to underpin the origins of complex chemistry is presented in Sect. 4, which is followed by the conclusions of this study.

\section{Observations and analysis}

\subsection{Observations}

The MYSOs NGC 7538 IRS9, W3 IRS5 , and AFGL490 located in Perseus NGC 7538 at $2.7 \mathrm{kpc}$, in Perseus W3 at $2.0 \mathrm{kpc}$, and in Camelopardalis $\mathrm{OB} 1$ at $1.4 \mathrm{kpc}$ respectively (see Table 1) were observed with the IRAM $30 \mathrm{~m}$ and the SMA. The three sources were observed with the IRAM $30 \mathrm{~m}$ telescope on February 19-20, 2012 using the EMIR $230 \mathrm{GHz}$ receiver and the new FTS backend. At these frequencies the IRAM $30 \mathrm{~m}$ beam is $\sim 10^{\prime \prime}$. The two sidebands cover $223-231 \mathrm{GHz}$ and 239-247 GHz at a spectral resolution of $\sim 0.2 \mathrm{~km} \mathrm{~s}^{-1}$ and with a sideband rejection of $-15 \mathrm{~dB}$ (Carter et al. 2012). We checked the pointing every one to two hours and found to be accurate within $2^{\prime \prime}$ to $3^{\prime \prime}$.

Focus was checked every four hours and generally remained stable through most of the observations; i.e., corrections in the range of $0.2-0.4$ were common, but a correction of 0.7 was required once. We acquired spectra in both position-switching and wobbler-switching modes. The resulting spectra had similar relative line intensities, indicative of no emission in the wobbler-off position. The wobbler-switching mode was considerably more stable, and we used these data alone for the quantitative analysis. The weather during the observations was excellent and the $\tau_{225 \mathrm{GHz}}$ varied between 0.05 and 0.15 . We converted the raw IRAM spectra to main beam temperatures and fluxes using forward and beam efficiencies and antenna temperature to flux

1 The Submillimeter Array is a joint project between the Smithsonian Astrophysical Observatory and the Academia Sinica Institute of Astronomy and Astrophysics. It is funded by the Smithsonian Institute and the Academia Sinica. 
Table 1. Source characteristics and ice abundances.

\begin{tabular}{lccccccccc}
\hline \hline \multirow{2}{*}{ Source } & \multirow{2}{*}{$\alpha(2000)$} & $\delta(2000)$ & \multicolumn{1}{c}{$L$} & \multicolumn{2}{c}{$N_{\mathrm{H}_{2} \mathrm{O}}$} & \multicolumn{3}{c}{$\mathrm{X}[\%]\left(/ N_{\mathrm{H}_{2} \mathrm{O}}\right)$} \\
\cline { 4 - 8 } & & & $\mathrm{kpc}$ & $10^{4} L_{\odot}$ & $10^{17} \mathrm{~cm}^{-2}$ & $\mathrm{CH}_{3} \mathrm{OH}$ & $\mathrm{CH}_{4}$ & $\mathrm{NH}_{3}$ & $\mathrm{OCN}^{-}$ \\
\hline NGC 7538 IRS9 & $23: 14: 01.6$ & $+61: 27: 20.4$ & 2.7 & 3.5 & 70 & $4.3 \pm 0.6$ & $2 \pm 0.4$ & $15 \pm 2.7$ & $1.7 \pm 0.5$ \\
W3 IRS5 & $02: 25: 40.5$ & $+62: 05: 51.3$ & 2.0 & 17 & 51 & $<3.3$ & $<1.3$ & $<5.7$ & $<0.23$ \\
AFGL490 & $03: 27: 38.7$ & $+58: 47: 01.1$ & 1.4 & 0.46 & 6.2 & $11 \pm 4$ & $<2.4$ & $<16$ & $<1.2$ \\
W33A & $18: 14: 38.9$ & $-17: 52: 04.0$ & 3.8 & 5.3 & 110 & $15 \pm 5$ & $1.5 \pm 0.2$ & $15 \pm 4$ & $6.3 \pm 1.9$ \\
AFGL2591 & $20: 29: 24.6$ & $+40: 11: 19.0$ & 3.3 & 18 & 12 & $14 \pm 2$ & $<2.7$ & $<2.3$ & - \\
NGC 7538 IRS1 & $23: 13: 45.4$ & $+61: 28: 12.0$ & 2.4 & 15 & 22 & $<4$ & $1.5 \pm 0.5$ & $<17$ & $<0.5$ \\
Orion IRc2 & $05: 35: 14.3$ & $-05: 22: 31.6$ & 0.4 & 1.0 & 24.5 & $10 \pm 3$ & - & - & $2 \pm 0.6$ \\
G24.78 & $18: 36: 12.6$ & $-07: 12: 11.0$ & 7.7 & 1.2 & - & - & - & - & - \\
G75.78 & $20: 21: 44.1$ & $+37: 26: 40.0$ & 1.9 & 19 & - & - & - & - & - \\
NGC 6334 IRS1 & $17: 20: 53.0$ & $-35: 47: 02.0$ & 1.7 & 11 & - & - & - & - & - \\
\hline
\end{tabular}

Notes. The sources observed in this study are in boldface, the others are from Bisschop et al. (2007).

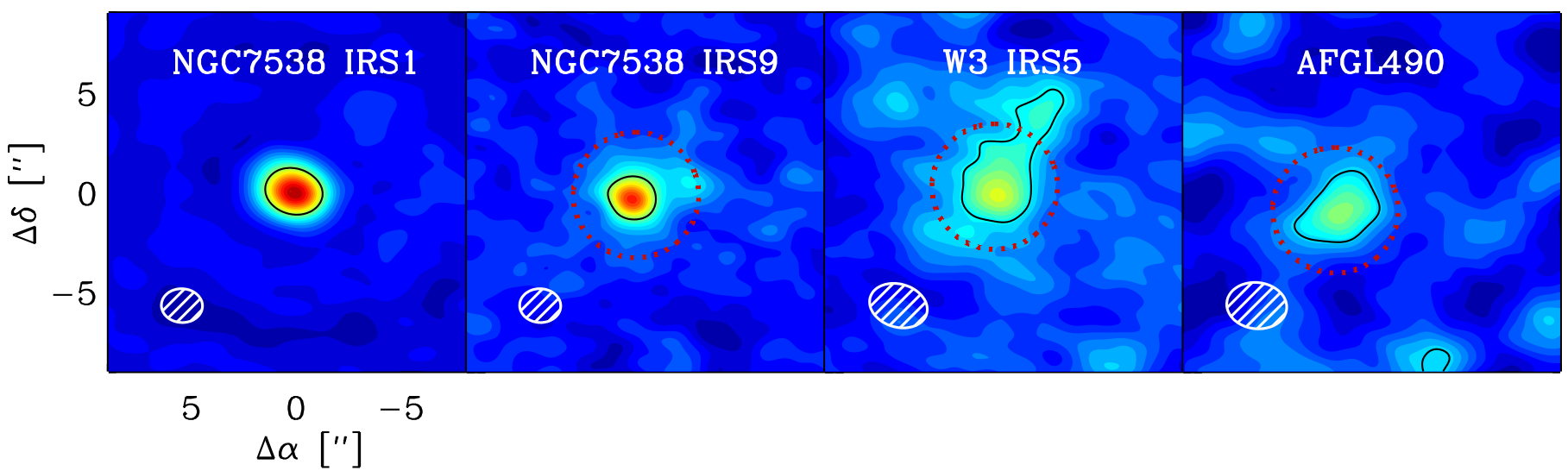

Fig. 1. Image of the $\mathrm{CH}_{3} \mathrm{CN}$ emission using the $13_{0}-12_{0}$ at $239.138 \mathrm{GHz}$ line acquired by the SMA for the massive young stellar objects NGC 7538 IRS9, W3 IRS5, and AFGL490 targeted in this study. The black contour presents the 50\% line intensity, and the synthesized beam is shown in white at the bottom left. A $2^{\prime \prime}$ radius mask used to extract the spectra is overplotted in dashed red line. Images for the hot core source NGC 7538 IRS1 is presented as well. The latter source has been through the same program as the three other sources.

conversion values ${ }^{2}$. The spectra were reduced using CLASS $^{3}$. A linear baseline was fitted to each $4 \mathrm{GHz}$ spectral chunk using four to seven windows. The individual scans were baselinesubtracted and averaged ${ }^{4}$. The absolute flux scale of the lines were then set using calibrated SMA data as outlined in detail by Öberg et al. (2013).

SMA observations were acquired in the compact and extended array configurations. The data in the compact configuration were taken on 15 October 2011 for all sources and with seven antennas, resulting in baselines between $16 \mathrm{~m}$ and $77 \mathrm{~m}$. The data in the extended configuration were obtained using eight antennas, resulting in $44 \mathrm{~m}$ to $226 \mathrm{~m}$ baselines and were acquired on 29 July 2011 for W3 IRS5 and AFGL490 and on the 15th of August 2011 for NGC 7538 IRS9. We set-up the SMA correlator to obtain a spectral resolution of $\sim 1 \mathrm{~km} \mathrm{~s}^{-1}$ using 128 channels for each of the 46 chunks covering $227-231 \mathrm{GHz}$ in the lower sideband and 239-243 GHz in the upper sideband. The $\tau_{225 \mathrm{GHz}}$ was 0.09 on 29 July, 0.1 on 15 August, and 0.07 on 15 October $2011^{5}$.

\footnotetext{
2 Listed at

WWW . iram.es/IRAMES/mainWiki/Iram30mEfficiencies

3 CLASS website:

http: //www . iram. fr/IRAMFR/GILDAS

4 Reduced data are available through the dataverse network at http://dx.doi.org/10.7910/DVN/26562

5 Observations are available on the SMA archive website

http://www.cfa.harvard.edu/cgi-bin/sma/smaarch.pl
}

We used the MIR package ${ }^{6}$ to perform the first data reduction steps (flux calibration and continuum subtraction). Absolute flux calibration is done with Callisto. The bandpass calibrators 1924-292 and 3c84 were used for the compact observations, and $3 \mathrm{c} 454.3$ and $3 \mathrm{c} 279$ were used to calibrate 29 July and 15 July observations, respectively. The quasars 0014+612 and $0102+584$ were used as gain calibrators for NGC 7538 IRS9, and $0244+624,0359+509$, and we used $0102+584$ for W3 IRS5 and AFGL490. The compact and extended data were combined for each source with MIRIAD ${ }^{6}$ using natural or robust weighting, depending on the data quality, which resulted in synthesized beam sizes of $2.0^{\prime \prime} \times 1.7^{\prime \prime}$ for NGC 7538 IRS9, $2.2^{\prime \prime} \times 2.8^{\prime \prime}$ for W3 IRS5, and 2.3" $\times 2.9^{\prime \prime}$ for AFGL490.

\subsection{Spectral extraction and rms}

Both the IRAM and SMA data were frequency-calibrated using the bright 5-4 $\mathrm{CH}_{3} \mathrm{OH}$ ladder around $241.7 \mathrm{GHz}$, correcting for the intrinsic velocity of the different sources. We extracted the SMA spectra using a $2^{\prime \prime}$-radius mask around the continuum phase center of each source. The mask dimension was chosen to encompass a majority of the $\mathrm{CH}_{3} \mathrm{CN}$ line emission at $239.318 \mathrm{GHz}$ that can be associated with a core component,

\footnotetext{
6 MIR website:

http://www.cfa.harvard. edu/ cqi/mircook.html
} 


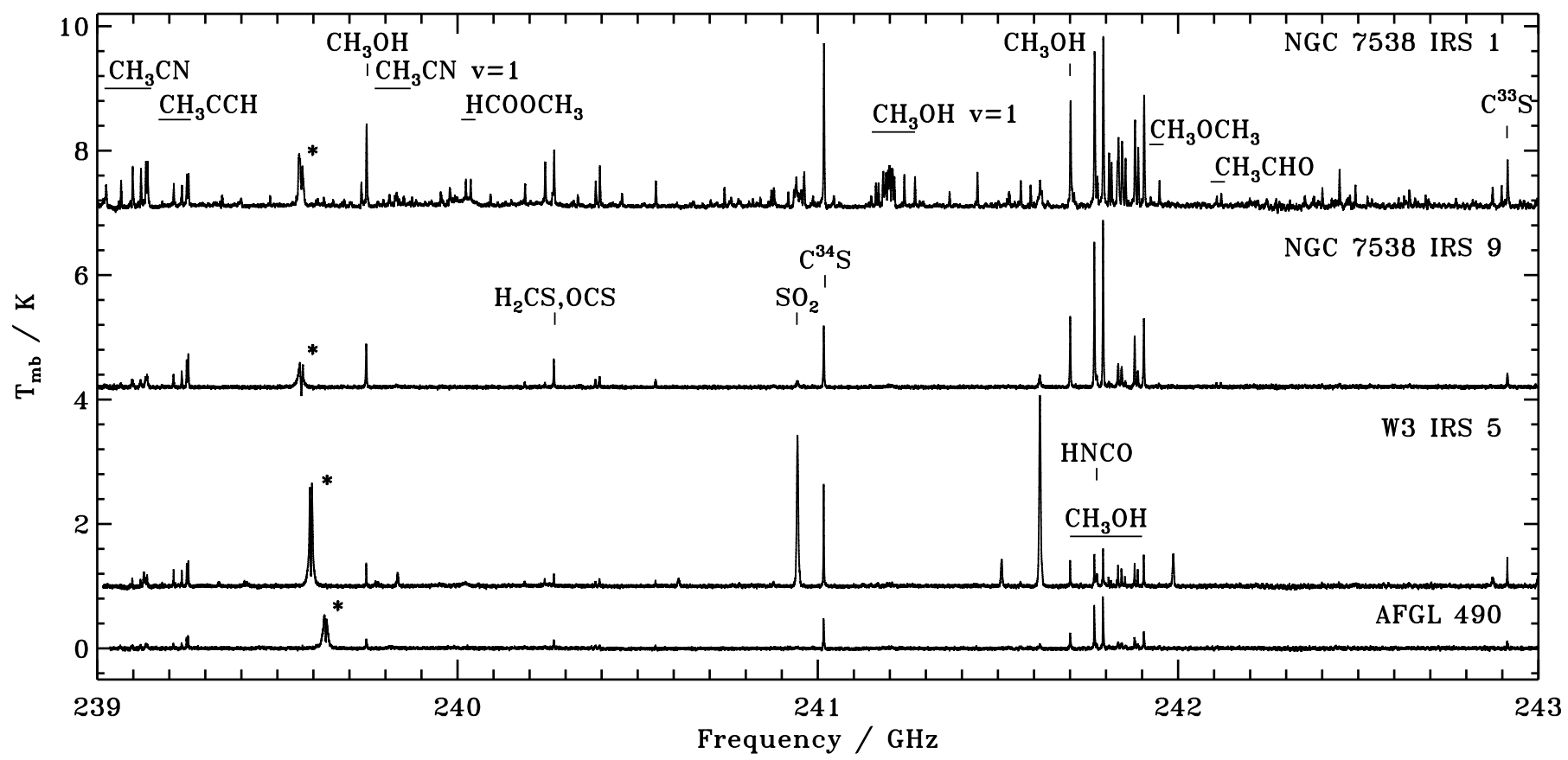

Fig. 2. 239-243 GHz spectral window from the IRAM $30 \mathrm{~m}$ displaying emission lines for typical hot core source NGC 7538 IRS1 and weak line MYSOs NGC 7538 IRS9, W3IRS5, AFGL490. The star-marked lines are CO ghost lines consistent with the sideband rejection for each source.

as shown in Fig. 1. We selected a $2^{\prime \prime}$ mask size based on a combination of theory and data inspection, i.e. the optimal mask size should include all the hot emission and exclude as much as possible of the cold envelope emission. In all sources, the selected mask size should be bigger than the $100 \mathrm{~K}$ radius and thus incorporate all emission associated with a potential hot core. Toward NGC 7538 IRS9 and AFGL 490, where the $100 \mathrm{~K}$ radius should be smaller than $2^{\prime \prime}$, smaller masks were also explored to more exclusively trace the $T>100 \mathrm{~K}$ region, but the resulting spectra had generally too low signal-to-noise ratio to be useful for a quantitative analysis. Some colder chemistry contribution to the SMA spectra in these sources cannot, thus, be excluded a priori, but the succeeding analysis (see below) demonstrated that the emission is indeed dominated by hot gas.

The rms for the IRAM and SMA observations of each source was derived in a line free region of several hundred channels: the 229.37-229.445 GHz region for the lower side band and the 240.7-240.75 GHz region for the upper side band. The rms derived for the IRAM observations is between 15 and $20 \mathrm{mK}$, which is lower than any previous millimeter observations for these sources. For the SMA data, the rms for the lower side band is $\sim 70 \mathrm{mK}$, and $100 \mathrm{mK}$ for the upper side band.

\section{Results}

\subsection{Line identification and characterization}

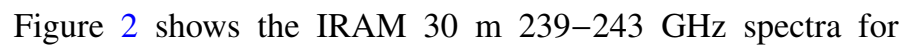
the three targeted line-poor MYSOs and the hot-core source NGC 7538 IRS1. The organic-poor MYSOs have, as expected, a lower line density, but also many line coincidences with the hot core. Of the lines from complex organics listed by Bisschop et al. (2007) found in their sample hot-core sources, $\mathrm{CH}_{3} \mathrm{OH}, \mathrm{CH}_{3} \mathrm{CN}$, $\mathrm{CH}_{3} \mathrm{CCH}, \mathrm{HNCO}, \mathrm{CH}_{3} \mathrm{OCH}_{3}$, and $\mathrm{CH}_{3} \mathrm{CHO}$ lines were identified in at least one of the organic-poor MYSOs using the splatalogue catalog tool ${ }^{7}$ and the $\mathrm{CDMS}^{8}$ and the JPL 9 spectral databases (Müller et al. 2001; Pickett et al. 1998). All available lines in the observed spectral range were used for the quantitative analysis except for $\mathrm{CH}_{3} \mathrm{OH}$ where we only used the lines from the 5-4 ladder to simplify the excitation analysis.

We fitted the identified lines with a Gaussian function in IDL using the routine "gaussfit" for isolated lines and "mpfitfun" when a multiple Gaussian fit was required because of overlapping lines. A local baseline component was added to the fits when needed, and the presented uncertainties were output by the fitting routines. We calculated $3 \sigma$ upper limits using an average FWHM for the different sources. Unresolved multiplets were treated in one out three ways depending on the nature of the overlapping lines: 1) if one of the possible contributing lines had a very low Einstein coefficient or high upper energy level and/or is not likely to be detected based on non-detections of the same species in other frequency ranges, then it was assumed to not contribute significantly and was not included in the fit. 2) If the lines came from the same species and the upper energy level and Einstein coefficients were identical or close to identical, then the degeneracies were added and the feature was treated as a single line; 3 ) if none of the two previous conditions were met, we did not include the multiplet in the analysis.

Line upper energy levels, Einstein coefficients, degeneracies, and quantum numbers from the Splatalogue are listed with the derived line fluxes and FWHM in Table 2 for $\mathrm{CH}_{3} \mathrm{OH}$ from the single-dish observations, in Table 3 for $\mathrm{CH}_{3} \mathrm{OH}$ from the SMA spectra, in Table 4 for $\mathrm{CH}_{3} \mathrm{CN}$ from IRAM, in Table 5 for $\mathrm{CH}_{3} \mathrm{CN}$ from the SMA, in Table 6 for $\mathrm{CH}_{3} \mathrm{CCH}$ from IRAM, in Table 7 for $\mathrm{CH}_{3} \mathrm{CCH}$ from the SMA, and in Table 8 for HNCO, $\mathrm{CH}_{3} \mathrm{OCH}_{3}$, and $\mathrm{CH}_{3} \mathrm{CHO}$. Only the lines with an Einstein coefficient logarithm higher than -4.5 and their upper level energy

\footnotetext{
7 Splatalogue website: http://www.cv.nrao.edu/php/splat/

8 CDMS website: http://www.astro.uni-koeln.de/cdms

9 JPL database website: http://spec.jpl .nasa.gov/
} 
E. C. Fayolle et al.: New constraints on the origins of organics

Table 2. $\mathrm{CH}_{3} \mathrm{OH}$ lines data from IRAM $30 \mathrm{~m}$ spectra.

\begin{tabular}{|c|c|c|c|c|c|c|c|c|c|c|}
\hline \multirow{2}{*}{$\begin{array}{l}\text { Freq. } \\
(\mathrm{GHz})\end{array}$} & \multirow{2}{*}{$\begin{array}{l}E_{\text {up }} \\
(\mathrm{K})\end{array}$} & \multirow[t]{2}{*}{$\log A$} & \multirow[t]{2}{*}{$g_{\mathrm{u}}$} & \multirow[t]{2}{*}{ Transition } & \multicolumn{2}{|c|}{ NGC 7538 IRS9 } & \multicolumn{2}{|c|}{ W3 IRS5 } & \multicolumn{2}{|c|}{ AFGL490 } \\
\hline & & & & & $\underset{\left(\mathrm{Jy} \mathrm{km} \mathrm{s}^{-1}\right)}{F \mathrm{~d} V}$ & $\begin{array}{c}F H W M \\
\left(\mathrm{~km} \mathrm{~s}^{-1}\right)\end{array}$ & $\underset{\left(\mathrm{Jy} \mathrm{km} \mathrm{s}^{-1}\right)}{\int F \mathrm{~d} V}$ & $\begin{array}{c}F H W M \\
\left(\mathrm{~km} \mathrm{~s}^{-1}\right)\end{array}$ & $\underset{\left(\mathrm{Jy} \mathrm{km} \mathrm{s}^{-1}\right)}{\int F \mathrm{~d} V}$ & $\begin{array}{c}F H W M \\
\left(\mathrm{~km} \mathrm{~s}^{-1}\right)\end{array}$ \\
\hline 239.746 & 49.1 & & 11 & $5_{1,5}-4_{1,4} \mathrm{~A}^{+}$ & $20.7 \pm 2.3$ & $3.70 \pm 0.03$ & $6.1 \pm 0.8$ & $2.32 \pm 0.04$ & $4.6 \pm 0.7$ & $4.4 \pm 0.2$ \\
\hline 241.700 & 47.9 & -4.22 & 11 & $5_{0,5}-4_{0,4} \mathrm{E}$ & $30.8 \pm$ & $3.47 \pm 0.02$ & $7.1 \pm 0.9$ & $2.43 \pm 0.04$ & $6.7 \pm 0.9$ & $3.53 \pm 0.07$ \\
\hline 241.767 & 40.4 & -4.24 & 11 & $5_{-1,5}-4_{-1,4} \mathrm{E}$ & $60.7 \pm 6.3$ & $3.37 \pm 0.01$ & $11.2 \pm 1.3$ & $2.98 \pm 0.03$ & $16.7 \pm 1.9$ & $3.11 \pm 0.02$ \\
\hline 241.791 & 34.8 & -4.22 & 11 & $5_{0,5}-4_{0,4} \mathrm{~A}$ & $69.2 \pm 7.1$ & $3.37 \pm 0.01$ & $12.6 \pm 1.5$ & $2.91 \pm 0.03$ & $20.3 \pm 2.2$ & $3.09 \pm 0.02$ \\
\hline 241.807 & 115.2 & -4.66 & 22 & $5_{4}-4_{4} \mathrm{~A}^{ \pm}$ & $2.3 \pm 0.5$ & $4.5 \pm 0.4$ & $1.6 \pm 0.3$ & $1.58 \pm 0.09$ & $<1.4$ & - \\
\hline 241.813 & 122.7 & -4.66 & 11 & $5-4,2-4-4,1 \mathrm{E}$ & $1.1 \pm 0.4$ & $3.6 \pm 0.6$ & $1.2 \pm 0.3$ & $1.7 \pm 0.2$ & $<1.4$ & - \\
\hline 241.830 & 130.8 & -4.66 & 11 & $5_{4,1}-4,0 \mathrm{E}$ & $<1.1$ & - & $1.3 \pm 0.3$ & $2.0 \pm 0.2$ & $<1.4$ & - \\
\hline 241.833 & 84.6 & -4.41 & 22 & $5_{3}-4_{3} \mathrm{~A}^{ \pm}$ & $13.3 \pm 1.6$ & $4.61 \pm 0.06$ & $5.5 \pm 0.7$ & $2.18 \pm 0.04$ & $3.6 \pm 0.6$ & $4.9 \pm 0.2$ \\
\hline 241.842 & 72.5 & -4.29 & 11 & $5_{2,4}-4_{2,3} \mathrm{~A}^{-}$ & $10.1 \pm 1.4$ & $5.5 \pm 0.1$ & $3.2 \pm 0.5$ & $1.90 \pm 0.08$ & $2.6 \pm 0.6$ & $6.8 \pm 0.5$ \\
\hline 241.844 & 82.5 & -4.41 & 11 & $5_{3,2}-4_{3,1} \mathrm{E}$ & $2.4 \pm 0.5$ & $3.3 \pm 0.3$ & $3.2 \pm 0.5$ & $2.2 \pm 0.1$ & $1.5 \pm 0.3$ & $3.50 \pm 0.02$ \\
\hline 241.852 & 97.5 & -4.41 & 11 & $5_{-3,3}-4_{-3,2} \mathrm{E}$ & $3.2 \pm 0.6$ & $5.5 \pm 0.4$ & $2.2 \pm 0.4$ & $2.1 \pm 0.1$ & $1.5 \pm 0.4$ & $6.5 \pm 0.8$ \\
\hline 241.879 & 55.9 & -4.22 & 11 & $5_{1,4}-4_{1,3} E$ & $23.5 \pm 2.6$ & $3.64 \pm 0.02$ & $6.7 \pm 0.9$ & $2.58 \pm 0.05$ & $5.2 \pm 0.7$ & $4.1 \pm 0.1$ \\
\hline 241.888 & 72.5 & -4.29 & 11 & $5_{2,3}-4_{2,2} \mathrm{~A}^{+}$ & $8.5 \pm 1.1$ & $4.28 \pm 0.09$ & $3.8 \pm 0.5$ & $2.04 \pm 0.06$ & $2.5 \pm 0.5$ & $5.2 \pm 0.3$ \\
\hline 241.904 & 60.7 & -4.29 & 11 & $5_{-2,4}-4_{-2,3} \mathrm{E}$ & $15.9 \pm 1.7$ & $3.66 \pm 0.02$ & $5.0 \pm 0.6$ & $2.57 \pm 0.03$ & $3.9 \pm 0.5$ & $3.85 \pm 0.07$ \\
\hline 241.905 & 57.1 & -4.30 & 11 & $5_{2,3}-4_{2,2} \mathrm{E}$ & $15.9 \pm 1.7$ & $3.66 \pm 0.02$ & $5.0 \pm 0.6$ & $2.57 \pm 0.03$ & $3.9 \pm 0.5$ & $3.85 \pm 0.07$ \\
\hline
\end{tabular}

Table 3. $\mathrm{CH}_{3} \mathrm{OH}$ lines extracted from SMA observations with a $2^{\prime \prime}$-radius mask.

\begin{tabular}{|c|c|c|c|c|c|c|c|c|c|c|}
\hline \multirow{2}{*}{$\begin{array}{l}\text { Freq. } \\
(\mathrm{GHz})\end{array}$} & \multirow{2}{*}{$\begin{array}{l}E_{\text {up }} \\
(\mathrm{K})\end{array}$} & \multirow[t]{2}{*}{$\log A$} & \multirow[t]{2}{*}{$g_{\mathrm{u}}$} & \multirow[t]{2}{*}{ Transition } & \multicolumn{2}{|c|}{ NGC 7538 IRS9 } & \multicolumn{2}{|c|}{ W3 IRS5 } & \multicolumn{2}{|c|}{ AFGL490 } \\
\hline & & & & & $\underset{\left(\mathrm{Jy} \mathrm{km} \mathrm{s}{ }^{-1}\right)}{\int F \mathrm{~d} V}$ & $\begin{array}{c}F H W M \\
\left(\mathrm{~km} \mathrm{~s}^{-1}\right)\end{array}$ & $\underset{\left(\mathrm{Jy} \mathrm{km} \mathrm{s}^{-1}\right)}{\int F \mathrm{~d} V}$ & $\begin{array}{c}F H W M \\
\left(\mathrm{~km} \mathrm{~s}^{-1}\right)\end{array}$ & $\underset{\left(\mathrm{Jy} \mathrm{km} \mathrm{s}{ }^{-1}\right)}{\int F \mathrm{~d} V}$ & $\begin{array}{c}F H W M \\
\left(\mathrm{~km} \mathrm{~s}^{-1}\right)\end{array}$ \\
\hline 239.746 & & & 11 & & $7.6 \pm 1.1$ & $4.6 \pm 0.2$ & $2.3 \pm 0.5$ & $1.9 \pm 0.2$ & $3.0 \pm 0.7$ & $4.6 \pm 0.5$ \\
\hline & & & 11 & $5_{0,5}-4_{0,4} \mathrm{E}$ & & & & & & \\
\hline 241. & & 4 & 11 & $5-1,5-4-1,4 \mathrm{E}$ & $8.2 \pm 1.1$ & 3.5 & $3.2 \pm 0.6$ & & $1.8 \pm 0.5$ & \\
\hline 241.7 & 34.8 & -4.22 & 11 & $5_{0,5}-4_{0,4} \mathrm{~A}$ & $7.1 \pm 1.0$ & $3.16=$ & $3.5 \pm 0.7$ & & $2.4 \pm 0.6$ & $4.9 \pm 0.7$ \\
\hline 241.807 & 115.2 & -4.66 & 22 & $5_{4}-4_{4} \mathrm{~A}^{ \pm}$ & $2.1 \pm 0.7$ & $6 \pm 1$ & $1.4 \pm 0.5$ & & $<1.1$ & - \\
\hline 241.813 & 122.7 & -4.66 & 11 & $5-4,2-4-4,1 \mathrm{E}$ & $1.0 \pm 0.4$ & $3.6 \pm 0.9$ & $1.0 \pm 0.5$ & $2.8 \pm 0.8$ & $<1.1$ & - \\
\hline 241.830 & 130.8 & -4.66 & 11 & $5_{4,1}-4,0 \mathrm{E}$ & $<0.8$ & - & $<1.1$ & - & $<1.1$ & - \\
\hline 241.833 & & -4.41 & 22 & $5_{3}-4_{3} \mathrm{~A}^{ \pm}$ & $6.4 \pm 1.1$ & $4.7 \pm 0.3$ & $3.1 \pm 0.7$ & $2.9 \pm 0.3$ & $1.8 \pm 0.6$ & $4.6 \pm 0.8$ \\
\hline 241.852 & 7.5 & -4.41 & 11 & $5_{-3,3}-4_{-3,2} \mathrm{E}$ & $1.9 \pm 0.6$ & $3.4 \pm$ & $1.4 \pm 0.5$ & $1.5 \pm$ & $<1.1$ & - \\
\hline 241.879 & 55.9 & -4.22 & 11 & $5_{1,4}-4_{1,3} E$ & $5.5 \pm 0.9$ & $3.4 \pm 0.2$ & $2.6 \pm 0.6$ & $2.3 \pm 0.2$ & $1.9 \pm 0.6$ & $4.7 \pm 0.7$ \\
\hline & 72.5 & -4.29 & 11 & $5_{2,3}-4_{2,2} \mathrm{~A}^{+}$ & $4.6 \pm 0.8$ & $5.2 \pm 0.3$ & $1.6 \pm 0.5$ & $1.6 \pm 0.2$ & $1.8 \pm 0.7$ & $7 \pm 2$ \\
\hline & 60.7 & -4.29 & 11 & $5-2,4-4_{-2,3} \mathrm{E}$ & $5.3 \pm 0.8$ & $4.5 \pm 0.1$ & $1.7 \pm 0.3$ & $2.2 \pm 0.2$ & $1.7 \pm 0.4$ & $5.2 \pm 0.5$ \\
\hline 241.905 & 57.1 & -4.30 & 11 & $5_{2,3}-4_{2,2} \mathrm{E}$ & $5.3 \pm 0.8$ & $4.5 \pm 0.1$ & $1.7 \pm 0.3$ & $2.2 \pm 0.2$ & $1.7 \pm 0.4$ & $5.2 \pm 0.5$ \\
\hline
\end{tabular}

Table 4. $\mathrm{CH}_{3} \mathrm{CN}$ lines data from IRAM $30 \mathrm{~m}$ spectra.

\begin{tabular}{lcccccccccc}
\hline \hline $\begin{array}{l}\text { Freq. } \\
(\mathrm{GHz})\end{array}$ & $\begin{array}{c}E_{\mathrm{up}} \\
(\mathrm{K})\end{array}$ & $\log A$ & $g_{\mathrm{u}}$ & Transition & \multicolumn{2}{c}{$\mathrm{NGC} 7538$ IRS9 } & \multicolumn{2}{c}{ W3 IRS5 } & \multicolumn{2}{c}{ AFGL490 } \\
& & & & $\begin{array}{c}\int F \mathrm{~d} V \\
\left.(\mathrm{Jy} \mathrm{km} \mathrm{s})^{-1}\right)\end{array}$ & $\begin{array}{c}F H W M \\
\left(\mathrm{~km} \mathrm{~s}^{-1}\right)\end{array}$ & $\begin{array}{c}\int F \mathrm{~d} V \\
\left(\mathrm{Jy} \mathrm{km} \mathrm{s}^{-1}\right)\end{array}$ & $\begin{array}{c}F H W M \\
\left(\mathrm{~km} \mathrm{~s}^{-1}\right)\end{array}$ & $\begin{array}{c}\int F \mathrm{~d} V \\
\left(\mathrm{Jy} \mathrm{km} \mathrm{s}^{-1}\right)\end{array}$ & $\begin{array}{c}F H W M \\
\left(\mathrm{~km} \mathrm{~s}^{-1}\right)\end{array}$ \\
\hline 239.023 & 258.9 & -3.00 & 54 & $13_{5}-12_{5}$ & $1.6 \pm 0.5$ & $9 \pm 2$ & $<1.0$ & - & $<1.5$ & - \\
239.064 & 194.6 & -2.97 & 54 & $13_{4}-12_{4}$ & $2.0 \pm 0.7$ & $9 \pm 2$ & $0.9 \pm 0.3$ & $6 \pm 2$ & $1.2 \pm 0.4$ & $6 \pm 2$ \\
239.096 & 144.6 & -2.95 & 108 & $13_{3}-12_{3}$ & $5.3 \pm 0.8$ & $6.0 \pm 0.3$ & $2.2 \pm 0.4$ & $2.2 \pm 0.2$ & $2.4 \pm 0.6$ & $8 \pm 1$ \\
239.120 & 108.9 & -2.94 & 54 & $13_{2}-12_{2}$ & $4.5 \pm 0.7$ & $5.7 \pm 0.3$ & $1.7 \pm 0.4$ & $2.8 \pm 0.3$ & $1.5 \pm 0.5$ & $6 \pm 2$ \\
239.133 & 87.5 & -2.93 & 54 & $13_{1}-12_{1}$ & $5.8 \pm 0.8$ & $4.6 \pm 0.2$ & $2.4 \pm 0.4$ & $2.4 \pm 0.2$ & $2.1 \pm 0.5$ & $4.9 \pm 0.5$ \\
239.138 & 80.3 & -2.93 & 54 & $13_{0}-12_{0}$ & $7.4 \pm 1.0$ & $5.2 \pm 0.2$ & $2.6 \pm 0.4$ & $2.1 \pm 0.2$ & $2.4 \pm 0.5$ & $5.4 \pm 0.5$ \\
\hline
\end{tabular}

Table 5. $\mathrm{CH}_{3} \mathrm{CN}$ lines data from SMA spectra.

\begin{tabular}{lcccccccccc}
\hline \hline $\begin{array}{l}\text { Freq. } \\
(\mathrm{GHz})\end{array}$ & $\begin{array}{l}E_{\mathrm{up}} \\
(\mathrm{K})\end{array}$ & $\log A$ & $g_{\mathrm{u}}$ & Transition & \multicolumn{2}{c}{$\mathrm{NGC} 7538 \mathrm{IRS} 9$} & \multicolumn{2}{c}{ W3 IRS5 } & \multicolumn{2}{c}{ AFGL490 } \\
& & & & $\begin{array}{c}\int F \mathrm{~d} V \\
(\mathrm{Jy} \mathrm{km} \mathrm{s})\end{array}$ & $\begin{array}{c}F H W M \\
\left(\mathrm{~km} \mathrm{~s}^{-1}\right)\end{array}$ & $\begin{array}{c}\int F \mathrm{~d} V \\
\left(\mathrm{Jy} \mathrm{km} \mathrm{s}^{-1}\right)\end{array}$ & $\begin{array}{c}F H W M \\
\left(\mathrm{~km} \mathrm{~s}^{-1}\right)\end{array}$ & $\begin{array}{c}\int F \mathrm{~d} V \\
\left(\mathrm{Jy} \mathrm{km} \mathrm{s}^{-1}\right)\end{array}$ & $\begin{array}{c}F H W M \\
\left(\mathrm{~km} \mathrm{~s}^{-1}\right)\end{array}$ \\
\hline 239.064 & 194.6 & -2.97 & 54 & $13_{4}-12_{4}$ & $2.9 \pm 0.9$ & $7.6 \pm 1.2$ & $<0.8$ & - & $<1.9$ & - \\
239.096 & 144.6 & -2.95 & 108 & $13_{3}-12_{3}$ & $5.1 \pm 1.0$ & $5.6 \pm 0.4$ & $2.9 \pm 0.9$ & $6.8 \pm 1.2$ & $<1.9$ & - \\
239.120 & 108.9 & -2.94 & 54 & $13_{2}-12_{2}$ & $4.4 \pm 1.1$ & $9.6 \pm 1.0$ & $1.2 \pm 0.5$ & $2.9 \pm 0.9$ & $1.8 \pm 0.8$ & $6 \pm 2$ \\
239.133 & 87.5 & -2.93 & 54 & $13_{1}-12_{1}$ & $5.6 \pm 1.3$ & $6.9 \pm 0.7$ & $3.1 \pm 0.6$ & $4.4 \pm 0.4$ & $1.8 \pm 0.9$ & $6 \pm 2$ \\
239.138 & 80.3 & -2.93 & 54 & $13_{0}-12_{0}$ & $5.2 \pm 1.2$ & $6.3 \pm 0.6$ & $2.1 \pm 0.5$ & $2.7 \pm 0.4$ & $1.8 \pm 0.9$ & $6 \pm 2$ \\
\hline
\end{tabular}


Table 6. $\mathrm{CH}_{3} \mathrm{CCH}$ lines data from IRAM $30 \mathrm{~m}$ spectra.

\begin{tabular}{|c|c|c|c|c|c|c|c|c|c|c|}
\hline \multirow{2}{*}{$\begin{array}{l}\text { Freq. } \\
(\mathrm{GHz})\end{array}$} & \multirow{2}{*}{$\begin{array}{l}E_{\text {up }} \\
(\mathrm{K})\end{array}$} & \multirow[t]{2}{*}{$\log A$} & \multirow[t]{2}{*}{$g_{u}$} & \multirow[t]{2}{*}{ Transition } & \multicolumn{2}{|c|}{ NGC 7538 IRS9 } & \multicolumn{2}{|c|}{ W3 IRS5 } & \multicolumn{2}{|c|}{ AFGL490 } \\
\hline & & & & & $\begin{array}{c}\left.\int_{(\mathrm{Jy} \mathrm{km} \mathrm{s}} F \mathrm{~d}\right) \\
.\end{array}$ & $\begin{array}{c}F H W M \\
\left(\mathrm{~km} \mathrm{~s}^{-1}\right) \\
\end{array}$ & $\underset{\left(\mathrm{Jy} \mathrm{km} \mathrm{s}^{-1}\right)}{F \mathrm{~d} V}$ & $\begin{array}{c}F H W M \\
\left(\mathrm{~km} \mathrm{~s}^{-1}\right) \\
\end{array}$ & $\begin{array}{c}\int_{(\mathrm{Jy} \mathrm{km} \mathrm{s}} F \mathrm{~d} V \\
.1\end{array}$ & $\begin{array}{c}F H W M \\
\left(\mathrm{~km} \mathrm{~s}^{-1}\right)\end{array}$ \\
\hline & & & 16 & & & - & $<0.7$ & - & $<1.0$ & - \\
\hline & & & 58 & & $1.0 \pm 0$ & $3.4 \pm 0$ & $0.9 \pm$ & $2.5 \pm 0.6$ & $<1.0$ & - \\
\hline & & -4.00 & 16 & & $4.7 \pm 0$ & $3.1 \pm$ & $4.0 \pm$ & $2.00 \pm$ & $1.5 \pm 0.3$ & $3.5 \pm 0.4$ \\
\hline 239.2 & & -4.85 & 58 & & $5.1 \pm 0$ & $2.71 \pm 0$ & $3.6 \pm 0.5$ & $1.98 \pm 0$ & $1.5 \pm 0.4$ & $3.0 \pm 0.3$ \\
\hline 239.248 & 93.3 & -4.84 & 58 & & $9.0 \pm 1.1$ & $2.92 \pm 0.05$ & $5.8 \pm 0.7$ & $2.2 \pm 0.05$ & $2.7 \pm 0.4$ & $2.3 \pm 0.1$ \\
\hline 239.252 & 86.1 & -4.84 & 58 & $14_{0}-13_{0}$ & $10.2 \pm 1.2$ & $2.74 \pm 0.04$ & $6.4 \pm 0.8$ & $2.12 \pm 0.04$ & $3.7 \pm 0.5$ & $2.9 \pm 0.1$ \\
\hline
\end{tabular}

Table 7. $\mathrm{CH}_{3} \mathrm{CCH}$ lines data from SMA spectra.

\begin{tabular}{lcccccccccc}
\hline \hline $\begin{array}{l}\text { Freq. } \\
(\mathrm{GHz})\end{array}$ & $\begin{array}{l}E_{\text {up }} \\
(\mathrm{K})\end{array}$ & $\log A$ & $g_{u}$ & Transition & \multicolumn{2}{c}{$\mathrm{NGC} 7538 \mathrm{IRS} 9$} & \multicolumn{2}{c}{ W3 IRS5 } & \multicolumn{2}{c}{ AFGL490 } \\
& & & & $\begin{array}{c}\int F \mathrm{~d} V \\
(\mathrm{Jy} \mathrm{km} \mathrm{s})\end{array}$ & $\begin{array}{c}F H W M \\
\left(\mathrm{~km} \mathrm{~s}^{-1}\right)\end{array}$ & $\begin{array}{c}\int F \mathrm{~d} V \\
\left.(\mathrm{Jy} \mathrm{km} \mathrm{s})^{-1}\right)\end{array}$ & $\begin{array}{c}F H W M \\
\left(\mathrm{~km} \mathrm{~s}^{-1}\right)\end{array}$ & $\begin{array}{c}\int F \mathrm{~d} V \\
\left(\mathrm{Jy} \mathrm{km} \mathrm{s}^{-1}\right)\end{array}$ & $\begin{array}{c}F H W M \\
\left(\mathrm{~km} \mathrm{~s}^{-1}\right)\end{array}$ \\
\hline 239.088 & 346.1 & -4.07 & 16 & $14_{6}-13_{6}$ & $<0.9$ & - & $<0.7$ & - & $<1.0$ & - \\
239.179 & 201.7 & -4.88 & 58 & $14_{4}-13_{4}$ & $<0.9$ & - & $0.9 \pm 0.3$ & $2.5 \pm 0.6$ & $<1.0$ & - \\
239.211 & 151.1 & -4.00 & 16 & $14_{3}-13_{3}$ & $1.8 \pm 0.6$ & $3.2 \pm 0.5$ & $<1.1$ & - & $1.0 \pm 0.6$ & $4.2 \pm 1.5$ \\
239.234 & 115.0 & -4.85 & 58 & $14_{2}-13_{2}$ & $1.3 \pm 0.5$ & $3.5 \pm 0.7$ & $<1.1$ & - & $<1.0$ & - \\
239.248 & 93.3 & -4.84 & 58 & $14_{1}-13_{1}$ & $2.3 \pm 0.7$ & $3.5 \pm 0.5$ & $<1.1$ & - & $<1.0$ & - \\
239.252 & 86.1 & -4.84 & 58 & $14_{0}-13_{0}$ & $2.4 \pm 0.7$ & $2.9 \pm 0.4$ & $<1.1$ & - & $1.6 \pm 0.8$ & $5.9 \pm 1.9$ \\
\hline
\end{tabular}

Table 8. $\mathrm{HNCO}, \mathrm{CH}_{3} \mathrm{CHO}$, and $\mathrm{CH}_{3} \mathrm{OCH}_{3}$ lines data from the IRAM $30 \mathrm{~m}$ spectra and $\mathrm{HNCO}$ line data from the SMA 2" radius compact region.

\begin{tabular}{|c|c|c|c|c|c|c|c|c|c|c|c|}
\hline \multirow[t]{2}{*}{ Species } & \multirow{2}{*}{$\begin{array}{l}\text { Freq. } \\
(\mathrm{GHz})\end{array}$} & \multirow{2}{*}{$\begin{array}{l}E_{\text {up }} \\
(\mathrm{K})\end{array}$} & \multirow[t]{2}{*}{$\log A$} & \multirow[t]{2}{*}{$g_{\mathrm{u}}$} & \multirow[t]{2}{*}{ Transition } & \multicolumn{2}{|c|}{ NGC 7538 IRS9 } & \multicolumn{2}{|c|}{ W3 IRS5 } & \multicolumn{2}{|c|}{ AFGL490 } \\
\hline & & & & & & $\begin{array}{c}\int F \mathrm{~d} V \\
\left(\mathrm{Jy} \mathrm{km} \mathrm{s} \mathrm{km}^{-1}\right)\end{array}$ & $\begin{array}{c}F H W M \\
\left(\mathrm{~km} \mathrm{~s}^{-1}\right)\end{array}$ & $\begin{array}{c}\int F \mathrm{~d} V \\
\left(\mathrm{Jy} \mathrm{km} \mathrm{s} \mathrm{km}^{-1}\right)\end{array}$ & $\begin{array}{c}F H W M \\
\left(\mathrm{~km} \mathrm{~s}^{-1}\right)\end{array}$ & $\begin{array}{c}\int F \mathrm{~d} V \\
\left(\mathrm{Jy} \mathrm{km} \mathrm{s}^{-1}\right)\end{array}$ & $\begin{array}{c}F H W M \\
\left(\mathrm{~km} \mathrm{~s}^{-1}\right)\end{array}$ \\
\hline $\mathrm{HNCO}$ & 240.876 & 112.6 & -3.72 & 23 & $11_{1,11}-10_{1,10}$ & $2.3 \pm 0.6$ & $13 \pm 2$ & $2.1 \pm 0.5$ & $5.5 \pm 0.5$ & $<1.0$ & - \\
\hline \multirow{4}{*}{ IRAM } & 241.704 & 239.9 & -3.74 & 23 & $11_{2,10}-10_{2,9}$ & $<1.1$ & - & $<1.1$ & - & $<1.0$ & - \\
\hline & 241.708 & 239.9 & -3.74 & 23 & $11_{2,9}-10_{2,8}$ & $<1.1$ & - & $<1.1$ & - & $<1.0$ & - \\
\hline & 241.774 & 69.6 & -3.71 & 23 & $11_{0,11}-10_{0,10}$ & $5.9 \pm 0.4$ & $2.3 \pm 0.3$ & $4.4 \pm 0.7$ & $3.8 \pm 0.2$ & $2.1 \pm 0.3$ & $1.7 \pm 0.4$ \\
\hline & 242.640 & 113.1 & -3.71 & 23 & $11_{1,10}-10_{1,9}$ & $<1.1$ & - & $2.5 \pm 0.6$ & $8.9 \pm 0.8$ & $<1.0$ & - \\
\hline $\mathrm{HNCO}$ & 240.876 & 112.6 & -3.72 & 23 & $11_{1,11}-10_{1,10}$ & $<1.1$ & - & $<1.1$ & - & $<1.0$ & - \\
\hline \multirow[t]{2}{*}{ SMA } & 241.774 & 69.6 & -3.71 & 23 & $11_{0,11}-10_{0,10}$ & $3.2 \pm 0.7$ & $5.8 \pm 0.6$ & $4.4 \pm 0.9$ & $5.2 \pm 0.4$ & $<1.0$ & - \\
\hline & 242.640 & 113.1 & -3.71 & 23 & $11_{1,10}-10_{1,9}$ & $1.8 \pm 0.6$ & $10 \pm 2$ & $<1.1$ & - & $<1.0$ & - \\
\hline \multirow[t]{4}{*}{$\mathrm{CH}_{3} \mathrm{OCH}_{3}$} & 225.599 & 69.8 & -3.88 & 450 & $12_{1,12}-11_{0,11}$ & $1.7 \pm 0.4$ & $4.2 \pm 0.4$ & $0.6 \pm 0.3$ & $2.6 \pm 0.6$ & $1.5 \pm 0.5$ & $8 \pm 2$ \\
\hline & 240.985 & 26.3 & -3.99 & 154 & $5_{3,3}-4_{2,2}$ & $<1.1$ & - & $<0.8$ & - & $<1.3$ & - \\
\hline & 241.529 & 26.3 & -3.99 & 198 & $5_{3,2}-4_{2,3}$ & $<1.1$ & - & $<0.8$ & - & $<1.1$ & - \\
\hline & 241.947 & 81.1 & -3.78 & 378 & $13_{1,13}-12_{0,12}$ & $1.1 \pm 0.4$ & $3.5 \pm 0.8$ & $0.4 \pm 0.1$ & $1.2 \pm 0.3$ & $1.3 \pm 0.5$ & $6 \pm 2$ \\
\hline \multirow[t]{12}{*}{$\mathrm{CH}_{3} \mathrm{CHO}$} & 223.650 & 72.3 & -3.41 & 50 & $12_{1,12}-11_{1,11} \mathrm{E}$ & $1.1 \pm 0.3$ & $2.8 \pm 0.3$ & $<0.9$ & - & $<1.0$ & - \\
\hline & 223.660 & 72.2 & -3.41 & 50 & $121,12-11_{1,11} \quad \mathrm{~A}$ & $1.4 \pm 0.3$ & $3.2 \pm 0.3$ & $<0.9$ & - & $<1.0$ & - \\
\hline & 226.552 & 71.4 & -3.39 & 50 & $12_{0,12}-11_{0,11} \mathrm{E}$ & $1.5 \pm 0.3$ & $2.9 \pm 0.3$ & $<0.9$ & - & $<1.0$ & - \\
\hline & 226.593 & 71.3 & -3.39 & 50 & $12_{0,12}-11_{0,11} \mathrm{~A}$ & $1.7 \pm 0.4$ & $3.5 \pm 0.3$ & $<0.9$ & - & $<1.0$ & - \\
\hline & 229.775 & 61.5 & -4.29 & 46 & $11_{1,11}-10_{0,10} \mathrm{~A}$ & $<1.0$ & - & $<0.9$ & - & $<1.0$ & - \\
\hline & 230.302 & 81.0 & -3.38 & 50 & $12_{2,11}-11_{2,10} \mathrm{~A}$ & $1.0 \pm 0.3$ & $2.5 \pm 0.3$ & $<0.9$ & - & $<1.0$ & - \\
\hline & 230.316 & 81.1 & -3.38 & 50 & $12_{2,11}-11_{2,10} \mathrm{E}$ & $1.0 \pm 0.2$ & $2.3 \pm 0.3$ & $<0.9$ & - & $<1.0$ & - \\
\hline & 242.106 & 83.9 & -3.30 & 54 & $13_{1,13}-12,1, \mathrm{E}$ & $1.1 \pm 0.4$ & $2.6 \pm 0.5$ & $<0.9$ & - & $<1.0$ & - \\
\hline & 242.118 & 83.8 & -3.30 & 54 & $13_{1,13}-12,12 \mathrm{~A}$ & $1.4 \pm 0.3$ & $3.7 \pm 0.5$ & $<0.9$ & - & $<1.0$ & - \\
\hline & 244.789 & 83.1 & -3.29 & 54 & $13_{0,13}-12_{0,12} \mathrm{E}$ & $1.2 \pm 0.3$ & $3.7 \pm 0.5$ & $<0.9$ & - & $<1.0$ & - \\
\hline & 244.832 & 83.1 & -3.29 & 54 & $13_{0,13}-12_{0,12} \mathrm{~A}$ & $1.2 \pm 0.5$ & $4.2 \pm 0.9$ & $<0.9$ & - & $<1.0$ & - \\
\hline & 244.854 & 72.3 & -4.19 & 50 & $12_{1,12}-11_{0,11} \mathrm{E}$ & $<1.0$ & - & $<0.9$ & - & $<1.0$ & - \\
\hline
\end{tabular}

below $400 \mathrm{~K}$ are displayed in the tables. Due to the high line density for $\mathrm{CH}_{3} \mathrm{OCH}_{3}$ and $\mathrm{CH}_{3} \mathrm{CHO}$, only the lines with an upper energy level below $200 \mathrm{~K}$ are shown for these species. No other complex molecules were detected toward any of the sources. For molecules with weak lines, we only used the IRAM data since the SMA observations have lower spectral resolution and signal-to-noise ratio.

\subsection{Spatial origin of the line emission}

Figures 3-5 present the line fluxes of key molecules from both the single-dish and SMA observations toward the three
MYSOs. The IRAM beam is 6.2 to 7.2 times larger than the SMA mask ((IRAM radius at 227-243 GHz: 5-5.4") ${ }^{2} /($ SMA mask radius: $\left.2^{\prime \prime}\right)^{2}$ ). That most emission lines in these figures do not display a factor of six or seven difference between the IRAM $30 \mathrm{~m}$ and SMA spectra demonstrates a non-uniform emission across the object. Some emission line fluxes, most notably $\mathrm{CH}_{3} \mathrm{CN}$, are similar (within a factor of two) between the IRAM $30 \mathrm{~m}$ and SMA spectra, indicating of a large contribution from unresolved emission at the source center. In contrast, little or no $\mathrm{CH}_{3} \mathrm{CCH}$ flux from the IRAM is recovered by the SMA, which indicates extended emission. The fact that some $\mathrm{CH}_{3} \mathrm{CCH}$ IRAM $30 \mathrm{~m}$ fluxes are more than $6.2-7.2 \times$ higher than 


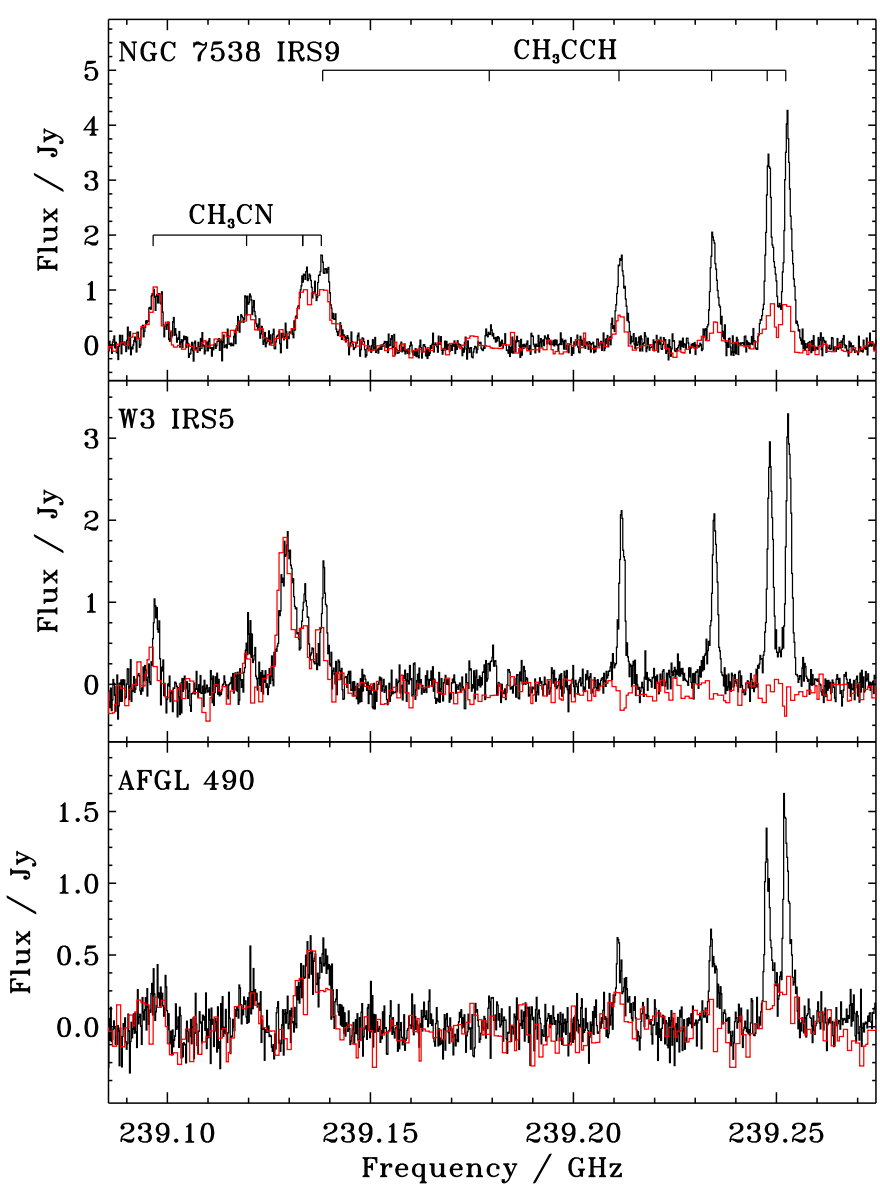

Fig. 3. Spectral window with several $\mathrm{CH}_{3} \mathrm{CN}$ and $\mathrm{CH}_{3} \mathrm{CCH}$ lines from the single-dish (black lines, $0.2 \mathrm{MHz}$ spectral resolution) and the $2 "$ interferometric data (red line, $0.8 \mathrm{MHz}$ spectral resolution).

the corresponding SMA fluxes is explained by spatial filtering of large-scale emission and/or off-centered emission. $\mathrm{CH}_{3} \mathrm{OH}$ lines display a mixed behavior: lines with higher upper energies show more overlap between the IRAM $30 \mathrm{~m}$ and SMA spectra than the colder lines. The IRAM $30 \mathrm{~m}$ and SMA line fluxes for the $11_{0,11}-10_{0,10} \mathrm{HNCO}$ line at $241.774 \mathrm{GHz}$ are close for NGC 7538 IRS9 and similar for W3 IRS5, but none of the IRAM 30 m flux is recovered by the AFGL490 SMA observations. Based on these lines, HNCO emission appears to be coming from both the core and the envelope of NGC 7538 IRS9, from the core of W3 IRS5 alone, and from the envelope of AFGL490. This source-to-source difference could partially come from different excitation conditions in the three sources, and the excitation-abundance structure degeneracy can only be strictly broken by observation of additional lines. The simplest scenario for explaining our detection is for HNCO to have both an extended and a compact origin, however, and this is also supported by the reported excitation characteristics and emission profile of $\mathrm{HNCO}$ in other sources (Bisschop et al. 2007).

In Fig. 5 the signal-to-noise ratio is lower, but it is still clear that $\mathrm{CH}_{3} \mathrm{CHO}$ toward NGC 7538 IRS9 only has extended emission since none of the IRAM $30 \mathrm{~m}$ line flux is recovered in the SMA spectra. No $\mathrm{CH}_{3} \mathrm{CHO}$ lines are detected in the other two MYSOs in the spectral range where IRAM $30 \mathrm{~m}$ and SMA observations overlap. $\mathrm{CH}_{3} \mathrm{OCH}_{3}$ is detected toward NGC 7538 IRS9 and AFGL490, and in both cases tentative SMA detections suggest that the emission originates in the source centers. Based on the different emission patterns, the molecules

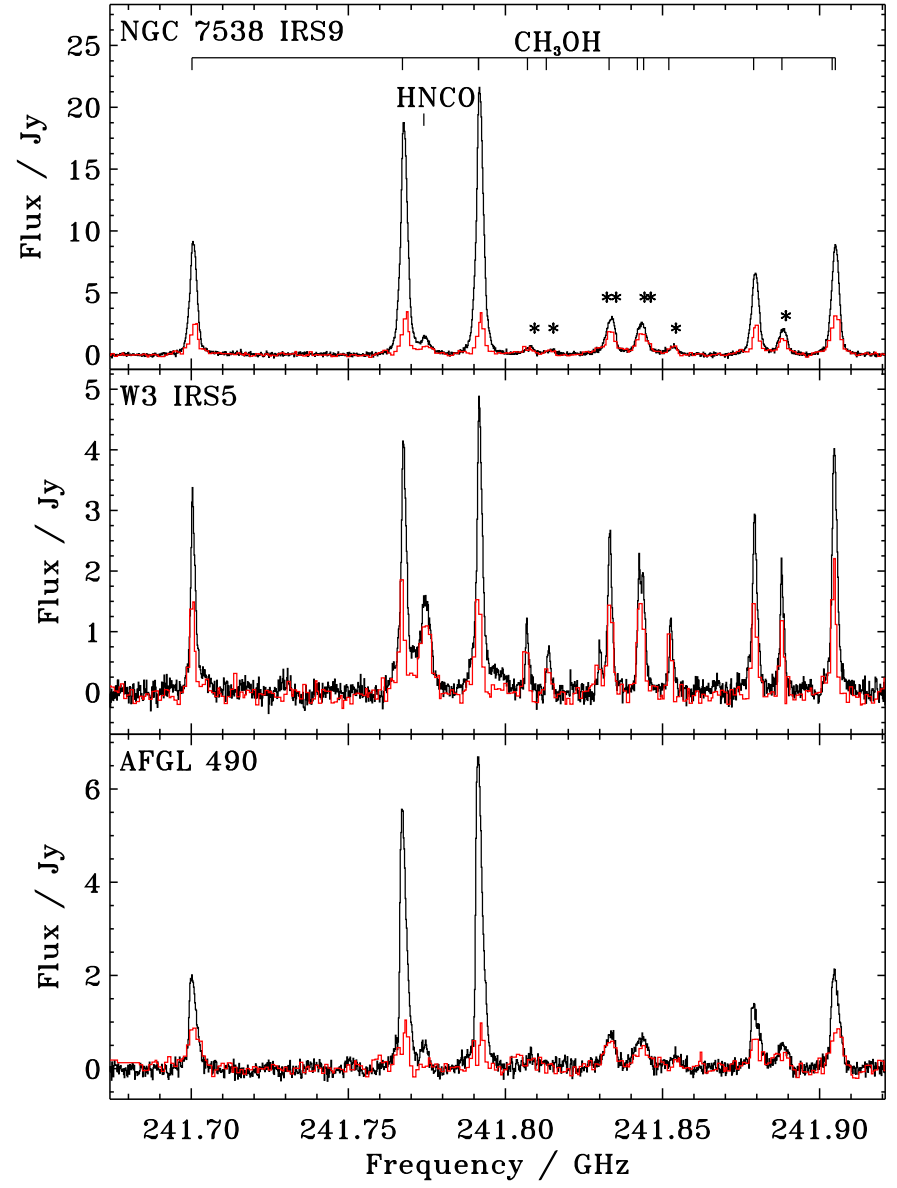

Fig. 4. Spectral window with several $\mathrm{CH}_{3} \mathrm{OH}$ lines from the singledish (black lines, $0.2 \mathrm{MHz}$ spectral resolution) and the 2" interferometric data (red line, $0.8 \mathrm{MHz}$ spectral resolution). $\mathrm{CH}_{3} \mathrm{OH}$ lines with upper level energies higher than $70 \mathrm{~K}$ are marked with a star in the NGC 7538 IRS9 to emphasize the increase in SMA/IRAM overlapping for lines with higher upper energy levels.

found in these spectra are classified as follows: $\mathrm{CH}_{3} \mathrm{CCH}$ and $\mathrm{CH}_{3} \mathrm{CHO}$ are envelope organics, $\mathrm{CH}_{3} \mathrm{CN}$ and $\mathrm{CH}_{3} \mathrm{OCH}_{3}$ are core organics, and $\mathrm{CH}_{3} \mathrm{OH}$ and $\mathrm{HNCO}$ are intermediate cases with significant core and envelope contributions.

\subsection{Rotational temperatures, and column densities}

The core and envelope classifications based on spatial emission patterns should be reflected in the rotational temperatures of the different molecules. Figure 6 shows the rotational diagrams for molecules with enough line detections, i.e., $\mathrm{CH}_{3} \mathrm{OH}$ (extracted from IRAM $30 \mathrm{~m}$ and SMA spectra) and $\mathrm{CH}_{3} \mathrm{CN}, \mathrm{CH}_{3} \mathrm{CCH}$, following the method described in Goldsmith \& Langer (1999). The line fluxes from the IRAM observations were converted into main beam temperature using the flux-to-antenna temperature conversion factor and the beam and forward efficiencies listed online for the EMIR receiver ${ }^{10}$ and linearly extrapolated for each line frequency $\left(T_{\mathrm{mb}}(\mathrm{K}) \simeq 0.2 \times \operatorname{Flux}(\mathrm{Jy})\right)$. The line fluxes from the SMA observation were converted into temperature using the Rayleigh-Jeans approximation with a circular beam of $2^{\prime \prime}$ radius coming from the mask dimension $\left(T_{\mathrm{mb}}(\mathrm{K}) \simeq\right.$ $1.3 \times \operatorname{Flux}(\mathrm{Jy}))$. Optically thin emission was assumed based on

\footnotetext{
10 http://wWw.iram.es/IRAMES/mainWiki/ Iram30 mEfficiencies
} 
Table 9. Rotational temperatures and column densities for $\mathrm{CH}_{3} \mathrm{OH}, \mathrm{CH}_{3} \mathrm{CN}$, and $\mathrm{CH}_{3} \mathrm{CCH}$ derived from the rotational diagrams presented in Fig. 6.

\begin{tabular}{|c|c|c|c|c|c|c|c|}
\hline \multirow[t]{2}{*}{ Species } & \multirow[t]{2}{*}{$B_{\mathrm{obs}} / B_{\text {emiss }}$} & \multicolumn{2}{|c|}{ NGC 7538 IRS9 } & \multicolumn{2}{|c|}{ W3 IRS5 } & \multicolumn{2}{|c|}{ AFGL490 } \\
\hline & & $T_{\text {rot }}(\mathrm{K})$ & $N\left(\mathrm{~cm}^{-2}\right)$ & $T_{\text {rot }}(\mathrm{K})$ & $N\left(\mathrm{~cm}^{-2}\right)$ & $T_{\text {rot }}(\mathrm{K})$ & $N\left(\mathrm{~cm}^{-2}\right)$ \\
\hline $\mathrm{CH}_{3} \mathrm{OH}$ (IRAM) & $5^{\prime \prime} / 5^{\prime \prime}$ & $25 \pm 2$ & $9 \pm 1 \times 10^{14}$ & & $3.2 \pm 0.4 \times 10^{14}$ & $25 \pm 2$ & $2.4 \pm 0.4 \times 10^{14}$ \\
\hline $\mathrm{CH}_{3} \mathrm{OH}$ (SMA) & $2^{\prime \prime} / 2^{\prime \prime}$ & $81 \pm 16$ & $2.5 \pm 0.4 \times 10^{15}$ & $166 \pm 85$ & $2.4 \pm 0.5 \times 10^{15}$ & $102 \pm 67$ & $1.2 \pm 0.5 \times 10^{15}$ \\
\hline $\mathrm{CH}_{3} \mathrm{CN}$ & $5^{\prime \prime} / 2^{\prime \prime}$ & $111 \pm 20$ & $7 \pm 2 \times 10^{13}$ & $92 \pm 23$ & $2.4 \pm 0.8 \times 10^{13}$ & $164 \pm 78$ & $3.6 \pm 1.2 \times 10^{13}$ \\
\hline $\mathrm{CH}_{3} \mathrm{CCH}$ & $5^{\prime \prime} / 5^{\prime \prime}$ & $47 \pm 5$ & $1.2 \pm 0.3 \times 10^{15}$ & $58 \pm 8$ & $7 \pm 2 \times 10^{14}$ & $41 \pm 7$ & $4.2 \pm 1.7 \times 10^{14}$ \\
\hline
\end{tabular}

Notes. $B_{\text {obs }}$ refers to the beam radius of the telescope used to obtain the molecular lines ( $5^{\prime \prime}$ for IRAM and $2^{\prime \prime}$ for SMA) and $B_{\text {emiss }}$ to the beam size where the line emission is assumed to be coming from.
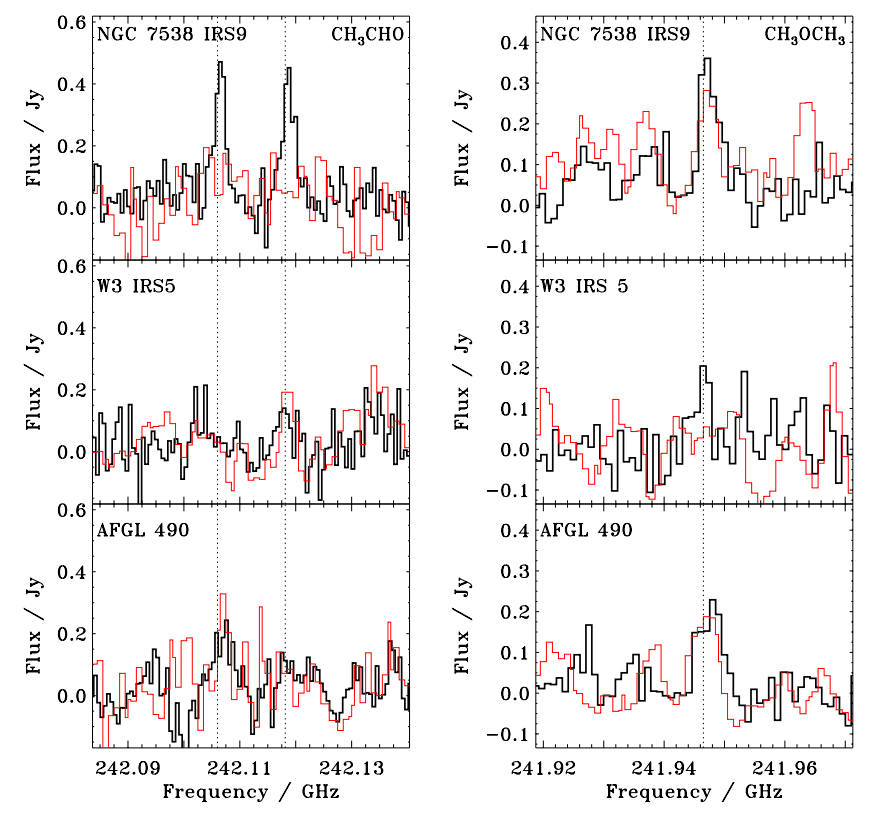

Fig. 5. Spectra of two $\mathrm{CH}_{3} \mathrm{CHO}$ lines at $242.106 \mathrm{GHz}$ and $242.118 \mathrm{GHz}$ (left panel) and the $\mathrm{CH}_{3} \mathrm{OCH}_{3}$ line at $241.946 \mathrm{GHz}$ (right panel) from the single-dish (black line, $0.2 \mathrm{MHz}$ spectral resolution) and 2" interferometric data (red line, $0.8 \mathrm{MHz}$ spectral resolution). The three line frequencies are marked by black dotted line.

the low line intensities and lack of asymmetry in the line profiles. This assumption was verified by the shape of the rotational diagram; i.e., flattening or large scatter was observed for lower energy transitions (cf., Bisschop et al. 2007). Considering the possibilities of subthermal excitation in the envelope, the rotational temperatures are not expected to be the gas kinetics temperatures outside of the core. A $10 \%$ uncertainty was added to the line-integrated area and is listed in the tables to account for the line shapes sometimes deviating from the Gaussian shape assumed for the fit. We used the "linfit" IDL routine to derive the rotational temperatures, as well as the column densities, and the routine returned the corresponding uncertainties.

Table 9 presents the column densities and rotational temperatures derived for these molecules using the rotational diagrams in Fig. 6. The beam-averaged $\mathrm{CH}_{3} \mathrm{OH}$ column densities and rotational temperatures derived from the IRAM $30 \mathrm{~m}$ spectra agree with those found by van der Tak et al. (2000), based on JCMT single-dish telescope at higher frequencies. The rotational temperature and column densities derived for $\mathrm{CH}_{3} \mathrm{OH}$ from the SMA data are always higher than those derived by the IRAM $30 \mathrm{~m}$, which is consistent with the SMA observations probing material closer to the MYSO centers. The E-/A- $\mathrm{CH}_{3} \mathrm{OH}$ ratio is consistent with unity within the uncertainties, which agrees with Wirström et al. (2011). The derived column densities for $\mathrm{CH}_{3} \mathrm{CN}$ from the IRAM data assume that the emission is only coming from the $2^{\prime \prime}$ radii encompassed by the SMA beam: i.e., we apply a dilution factor of 0.16 to account for the SMA extraction mask area ( $2^{\prime \prime}$ radius) to IRAM beam ( $5^{\prime \prime}$ radius) ratio. This assumption is justified by the $\mathrm{CH}_{3} \mathrm{CN}$ hot-core like rotational temperatures of $80-110 \mathrm{~K}$ toward the different MYSOs, which are also consistent with the $\mathrm{CH}_{3} \mathrm{OH}$ excitation temperature derived from the SMA spectra. It is also consistent with the observed overlap between the IRAM and SMA line fluxes (see Fig. 3). The rotational temperatures of $\sim 50 \mathrm{~K}$ obtained for the $\mathrm{CH}_{3} \mathrm{CCH}$ 14-13 ladder from the IRAM spectra are consistent with an envelope origin, but suggests that it is mainly present in the luke-warm envelope regions rather than in the outermost cold envelope.

The rotational diagram method assumes that all data can be described by a single excitation temperature. To test this assumption for our data, a two-temperature fit was explored for the case of methanol. Two-temperature fits of $\mathrm{CH}_{3} \mathrm{OH}$ lines was investigated by van der Tak et al. (2000), Leurini et al. (2007), and Isokoski et al. (2013), among others. The full results are presented in Appendix A, but briefly: both a cold and warm component are recovered from the IRAM $30 \mathrm{~m}$ data. The derived column densities of the cold components are consistent with the single-component fits (within uncertainties), while the warm component is consistent with the single-component fit to the SMA data. The fit to the SMA line data was not improved by adding a second component, verifying our hypothesis that the $2^{\prime \prime}$ mask emission is dominated by a hot component for all sources.

For $\mathrm{HNCO}, \mathrm{CH}_{3} \mathrm{CHO}$, and $\mathrm{CH}_{3} \mathrm{OCH}_{3}$, no rotational diagrams could be built owing to the very small upper-level energy range of the observed transitions, and column densities were calculated using the envelope temperature (the $\mathrm{CH}_{3} \mathrm{OH}$ IRAM $30 \mathrm{~m}$ rotational temperature) if the molecule was classified as an envelope molecule and the core temperature (the $\mathrm{CH}_{3} \mathrm{CN}$ rotational temperature) if the molecule was classified as a core molecule, and both rotational excitation temperatures if the molecule was classified as intermediate, i.e. HNCO. As seen in Table 10, the calculated $\mathrm{HNCO}$ abundance with respect to $\mathrm{CH}_{3} \mathrm{OH}$ is almost identical regardless of the assumed spatial origin of the line emission. For core molecules, the same dilution factor as for $\mathrm{CH}_{3} \mathrm{CN}$ was applied (see Table 10). For molecules with multiple line detections, the column densities were derived by averaging the individual column densities found for each detected line and taking the square root of the sum of the individual uncertainties squared as uncertainty. Only the IRAM data were used to calculate these column densities since these data present a higher signal-to-noise ratio. 
E. C. Fayolle et al.: New constraints on the origins of organics

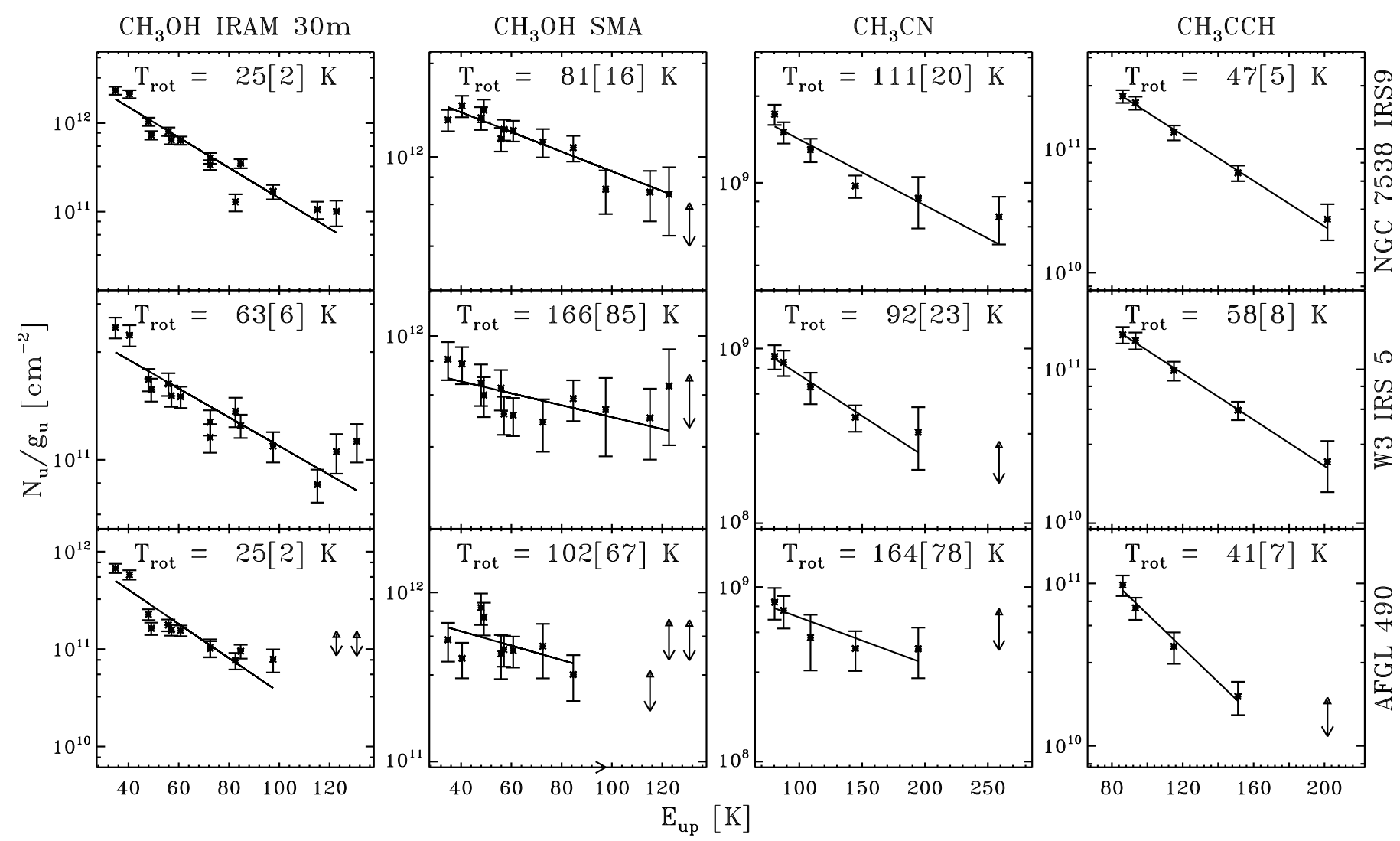

Fig. 6. Rotational diagrams of $\mathrm{CH}_{3} \mathrm{OH}$ from the single dish data (first column), for $\mathrm{CH}_{3} \mathrm{OH}$ from the SMA spectra extracted with a 2 " mask (second column), and $\mathrm{CH}_{3} \mathrm{CN}$ from the single-dish data (third column), and $\mathrm{CH}_{3} \mathrm{CCH}$ (fourth column) and for the sources NGC 7538 IRS9 (first row), W3 IRS5 (second row), and AFGL490 (third row).

Table 10. Column densities for $\mathrm{HNCO}, \mathrm{CH}_{3} \mathrm{CHO}, \mathrm{CH}_{3} \mathrm{OCH}_{3}$ using excitation temperatures from Table 7.

\begin{tabular}{lcccc}
\hline \hline Species & $B_{\text {obs }} / B_{\text {emiss }}$ & $\begin{array}{c}\text { NGC 7538 IRS9 } \\
N\left(\mathrm{~cm}^{-2}\right)\end{array}$ & $\begin{array}{c}\text { W3 IRS5 } \\
N\left(\mathrm{~cm}^{-2}\right)\end{array}$ & $\begin{array}{c}\text { AFGL490 } \\
N\left(\mathrm{~cm}^{-2}\right)\end{array}$ \\
\hline $\mathrm{HNCO}_{\text {ext }}$ & $5^{\prime \prime} / 5^{\prime \prime}$ & $4.0 \pm 1.3 \times 10^{13}$ & $1.8 \pm 0.3 \times 10^{13}$ & $6.0 \pm 2.2 \times 10^{12}$ \\
$\mathrm{HNCO}_{\text {comp }}$ & $5^{\prime \prime} / 2^{\prime \prime}$ & $1.1 \pm 0.3 \times 10^{14}$ & $1.4 \pm 0.6 \times 10^{14}$ & $<4 \times 10^{13}$ \\
$\mathrm{CH}_{3} \mathrm{OCH}$ & $5^{\prime \prime} / 2^{\prime \prime}$ & $3.3 \pm 1.0 \times 10^{14}$ & $9.7 \pm 4 \times 10^{13}$ & $4.5 \pm 1.9 \times 10^{14}$ \\
$\mathrm{CH}_{3} \mathrm{CHO}$ & $5^{\prime \prime} / 5^{\prime \prime}$ & $3.1 \pm 0.4 \times 10^{13}$ & $<1.1 \times 10^{13}$ & $<1.3 \times 10^{13}$ \\
\hline
\end{tabular}

Notes. $B_{\text {obs }}$ refers to the beam size of the telescope used to obtain the molecular lines ( $5^{\prime \prime}$ for IRAM and $2^{\prime \prime}$ for SMA) and $B_{\text {emiss }}$ to the beam size from where the line emission is assumed to be coming from.

Table 11. $\mathrm{CH}_{3} \mathrm{CN}, \mathrm{CH}_{3} \mathrm{CCH}, \mathrm{HNCO}, \mathrm{CH}_{3} \mathrm{CHO}, \mathrm{CH}_{3} \mathrm{OCH}_{3}$ abundances with respect to $\mathrm{CH}_{3} \mathrm{OH}$ for their respective spatial origin.

\begin{tabular}{lcccc}
\hline \hline $\begin{array}{l}\text { Species ratio } \\
/ \mathrm{CH}_{3} \mathrm{OH}\end{array}$ & Spatial origin & NGC 7538 IRS9 & W3 IRS5 & AFGL490 \\
\hline $\mathrm{CH}_{3} \mathrm{CN}$ & compact & $2.8 \pm 0.9 \times 10^{-2}$ & $1.0 \pm 0.4 \times 10^{-2}$ & $3.0 \pm 1.6 \times 10^{-2}$ \\
$\mathrm{CH}_{3} \mathrm{CCH}$ & extended & $1.3 \pm 0.4$ & $2.2 \pm 0.7$ & $1.8 \pm 0.8$ \\
$\mathrm{HNCO}$ & extended & $4.4 \pm 1.6 \times 10^{-2}$ & $5.6 \pm 1.2 \times 10^{-2}$ & $2.5 \pm 1.1 \times 10^{-2}$ \\
$\mathrm{HNCO}$ & compact & $4.4 \pm 1.4 \times 10^{-2}$ & $5.8 \pm 2.7 \times 10^{-2}$ & $<3.4 \times 10^{-2}$ \\
$\mathrm{CH}_{3} \mathrm{OCH}$ & compact & $1.3 \pm 0.5 \times 10^{-1}$ & $4.0 \pm 1.8 \times 10^{-2}$ & $3.8 \pm 2.3 \times 10^{-2}$ \\
$\mathrm{CH}_{3} \mathrm{CHO}$ & extended & $3.4 \pm 0.6 \times 10^{-2}$ & $<3.5 \times 10^{-2}$ & $<5.5 \times 10^{-2}$ \\
\hline
\end{tabular}

\subsection{Organics in hot cores vs. organic-poor MYSOs}

By definition, the organic-poor MYSOs reported in this study have less intense emission of complex organic molecules than do line-rich hot cores. The question for this section is whether the chemical composition with respect to $\mathrm{CH}_{3} \mathrm{OH}$ is different between the two source families. The $\mathrm{CH}_{3} \mathrm{CN}, \mathrm{CH}_{3} \mathrm{CCH}$, $\mathrm{HNCO}, \mathrm{CH}_{3} \mathrm{CHO}$, and $\mathrm{CH}_{3} \mathrm{OCH}_{3}$ abundances with respect to $\mathrm{CH}_{3} \mathrm{OH}$ obtained here and presented in Fig. 11 for the three MYSOs are compared to the hot core abundances derived by Bisschop et al. (2007) in Fig. 8. Moreover, three high-mass protostellar objects from Isokoski et al. (2013) have been added. 

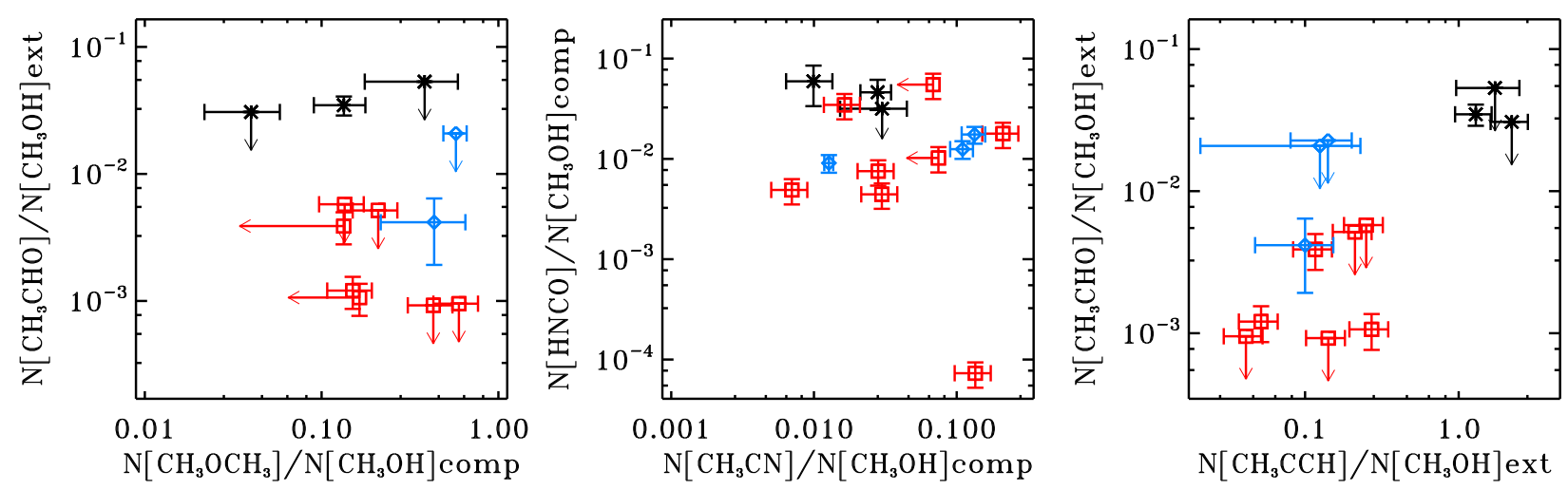

Fig. 7. Gas abundance correlation between organics including upper limits. The black crosses are the abundances derived for the MYSOs, the red squares are derived for hot-core sources by Bisschop et al. (2007), and the blue diamonds are results for hot-core sources from Isokoski et al. (2013). An arbitrary error of $20 \%$ has been taken when not reported in the two latest studies.

These three objects were inferred to have large equatorial structures, but Isokoski et al. (2013) found no strong chemical differences in their chemistry compared to the Bisschop et al. (2007) hot-core sources. For the three MYSOs sources, the molecular abundances with respect to $\mathrm{CH}_{3} \mathrm{OH}$ are calculated using the $\mathrm{CH}_{3} \mathrm{OH}$ column densities derived for the envelope if the molecule has been classified as "envelope" molecules and using the $\mathrm{CH}_{3} \mathrm{OH}$ column density derived for the core (SMA-based) in the case of a core molecule. For the hot core sources, Bisschop et al. (2007) applied a dilution factor corresponding to the region where $T>100 \mathrm{~K}$ for $\mathrm{CH}_{3} \mathrm{OH}, \mathrm{CH}_{3} \mathrm{CN}, \mathrm{HNCO}$, and $\mathrm{CH}_{3} \mathrm{OCH}_{3}$, but not for $\mathrm{CH}_{3} \mathrm{CCH}$ and $\mathrm{CH}_{3} \mathrm{CHO}$. To calculate $\mathrm{CH}_{3} \mathrm{CCH}$ and $\mathrm{CH}_{3} \mathrm{CHO}$ abundances with respect to $\mathrm{CH}_{3} \mathrm{OH}$, we removed the dilution factor for $\mathrm{CH}_{3} \mathrm{OH}$ applied by Bisschop et al. (2007). All other abundances were taken directly from Bisschop et al. (2007). Isokoski et al. (2013) could identify a cold and hot methanol emission using single-dish data and the derived column densities for both components have been used in Fig. 7 in the same way as for the sources analyzed in the present study.

The histograms in Fig. 8 show that the $\mathrm{CH}_{3} \mathrm{CN}, \mathrm{CH}_{3} \mathrm{OCH}_{3}$, and $\mathrm{HNCO}$ core abundances with respect to $\mathrm{CH}_{3} \mathrm{OH}$ are similar for the organic-poor MYSOs and the hot-core sources. In contrast, the organic-poor MYSOs show higher complex organic envelope abundances, i.e., $\mathrm{CH}_{3} \mathrm{CHO}$ and $\mathrm{CH}_{3} \mathrm{CCH}$, with respect to $\mathrm{CH}_{3} \mathrm{OH}$ compared to the hot core sources. This difference is most likely due to to our not being able to separate $\mathrm{CH}_{3} \mathrm{OH}$ core and envelope emission in the study by Bisschop et al. (2007), resulting in artificially low envelope ratios with respect to $\mathrm{CH}_{3} \mathrm{OH}$ when all $\mathrm{CH}_{3} \mathrm{OH}$ is implicitly assumed to originate in the envelope; in reality, the high excitation temperature of $\mathrm{CH}_{3} \mathrm{OH}$ in the hot core sources suggests that most of it really comes from the core.

A similar apparent separation between hot core and organicpoor MYSOs are visible in $\log -\log$ correlations of molecular abundances with respect to $\mathrm{CH}_{3} \mathrm{OH}$ shown in Fig. 7. Furthermore, there is a clear correlation between envelope molecules $\mathrm{CH}_{3} \mathrm{CHO}$ and $\mathrm{CH}_{3} \mathrm{CCH}$, but this may simply be due to the different abundance derivations of cold molecules with respect to $\mathrm{CH}_{3} \mathrm{OH}$ for the hot cores and the weak-line MYSOs, rather than signifying a chemical relationship. More interestingly, these $\log -\log$ abundance ratio plots show that there is no correlation between the two N-bearing organics $\mathrm{CH}_{3} \mathrm{CN}$ and $\mathrm{HNCO}$ over an order of magnitude range. There is also no correlation between the two O-bearing complex species $\mathrm{CH}_{3} \mathrm{OCH}_{3}$ and $\mathrm{CH}_{3} \mathrm{CHO}$, which is consistent with their inferred different origins in the organic-poor MYSOs.
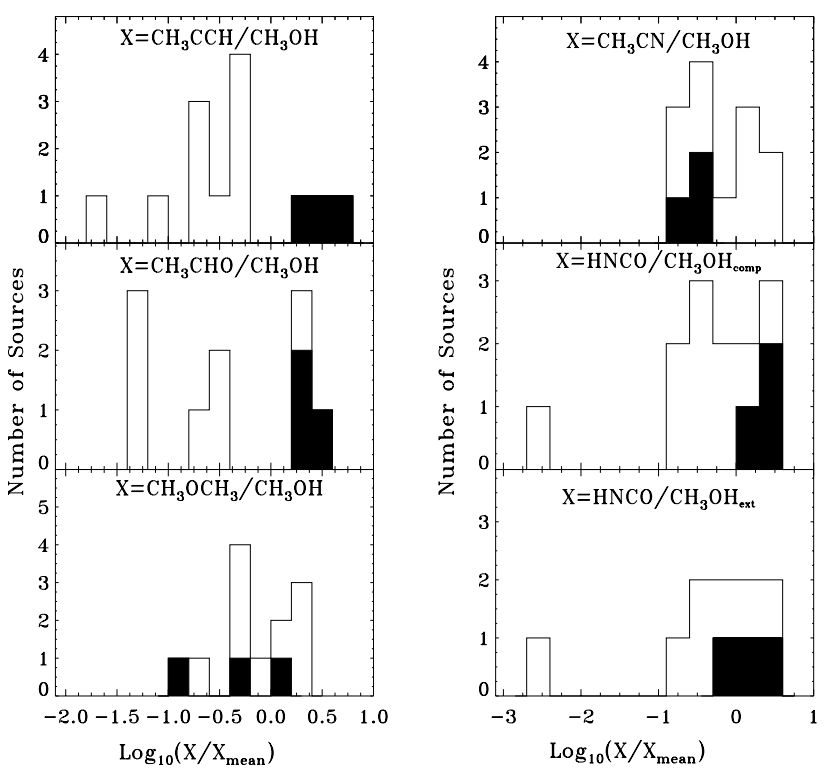

Fig. 8. Number of sources versus the logarithm of their gas phase organic ratio over methanol with respect to the mean for O-bearing species. The solid filled histograms correspond to sources observed and analyzed here, the unfilled histograms correspond to sources from Bisschop et al. (2007) and Isokoski et al. (2013). The left panel presents the O-bearing species data, while the right panel focuses on the N-bearing molecules. HNCO abundances were derived assuming either hot compact emission or cold extended emission, since its origin does not seem to be consistent between sources.

\subsection{An ice-gas connection?}

The $\mathrm{CH}_{3} \mathrm{OH}$ ice content may be an important factor in whether a hot core chemistry developed, so we compare the $\mathrm{CH}_{3} \mathrm{OH}$ core column density toward our organic-poor MYSOs and hot cores with their $\mathrm{CH}_{3} \mathrm{OH}$ ice abundance (with respect to $\mathrm{H}_{2} \mathrm{O}$ ) (see Table 1, Gibb et al. 2004). In the hot cores, the majority of the $\mathrm{CH}_{3} \mathrm{OH}$ gas originates in the core and we use the derived column densities from Bisschop et al. (2007), where all $\mathrm{CH}_{3} \mathrm{OH}$ emission is assumed to originate in the central region where the temperature is higher than $100 \mathrm{~K}$. To ensure a fair comparison we calculated the size of the "hot core region" toward our sample using the relation between luminosity and temperature $R_{T=100 \mathrm{~K}} \approx 2.3 \times 10^{14}\left(\sqrt{L / L_{\odot}}\right)$, which was shown by Bisschop et al. (2007) to approximate the $100 \mathrm{~K}$ radius well toward their 
E. C. Fayolle et al.: New constraints on the origins of organics

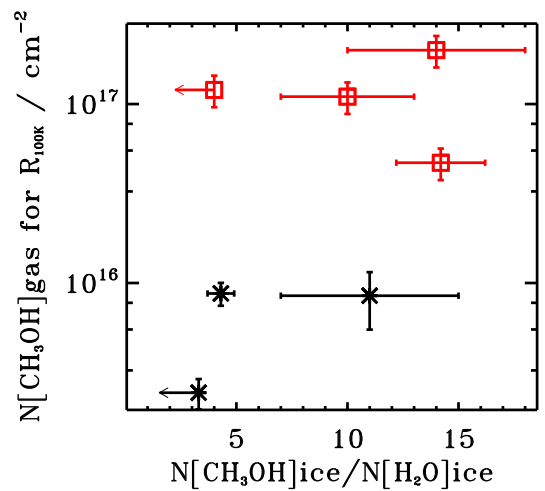

Fig. 9. $\mathrm{CH}_{3} \mathrm{OH}$ column density in the inner core (calculated area where $T>100 \mathrm{~K}$ ) versus $\mathrm{CH}_{3} \mathrm{OH}$ ice abundance over $\mathrm{H}_{2} \mathrm{O}$ ice in the envelope. The stars present the line-poor sources analyzed in this study, while the empty squares are data from Bisschop et al. (2007) for hot-core sources.

source sample. We then assumed that all $\mathrm{SMA} \mathrm{CH}_{3} \mathrm{OH}$ line flux originate in these regions, based on the derived rotational temperatures, and used an appropriate dilution factor when the $100 \mathrm{~K}$ area is smaller than the $2^{\prime \prime}$ mask used for spectral extraction. Figure 9 presents the resulting column density of hot $\mathrm{CH}_{3} \mathrm{OH}$ gas versus the initial $\mathrm{CH}_{3} \mathrm{OH}$ abundance on the grains. It appears that it is primarily the column density of $\mathrm{CH}_{3} \mathrm{OH}$ that is different between the line-rich and line-poor sources. No strong correlation with the ice content is observed, but more sources would allow the sample to be divided into luminosity and total mass bins, removing scatter due to initial physical conditions and size of $100 \mathrm{~K}$ region. Still, this plot suggests that initial $\mathrm{CH}_{3} \mathrm{OH}$ ice content alone does not determine the richness of the MYSO chemistry when correcting for the source luminosity.

The initial ice composition may also affect the complex organic composition in both hot cores and organic-poor MYSOs cores and envelopes. Figure 10 presents correlation plots between ratios of the $\mathrm{N}$-bearing organics and $\mathrm{CH}_{3} \mathrm{OH}$ in the ice and gas phases. The two gas-phase $\mathrm{N}$-bearing organics are $\mathrm{HNCO}$ and $\mathrm{CH}_{3} \mathrm{CN}$, and the two ice species are $\mathrm{OCN}^{-}$and $\mathrm{NH}_{3}$. The ice abundances are listed in Table 1 and have been obtained by Gibb et al. (2004). For sake of consistency, only the abundances obtained through the analysis technique described and performed by Gibb et al. (2004) were used here though detailed analysis of specific ice species, taking ice environment and using multiple vibrational bands into account, have been conduced, e.g., by Taban et al. (2003) in the case of $\mathrm{NH}_{3}$ in W 33A.

When combining our new observations with data from the literature, a sample of seven MYSOs have both ice and gas observations. As seen in Fig. 9, only a fraction of them can be used to correlate specific ice ratios, however, because of multiple ice abundance upper limits for many of the sources. For example, W3 IRS5 is not included in any of the plots, because of its $\mathrm{CH}_{3} \mathrm{OH}, \mathrm{OCN}^{-}$and $\mathrm{NH}_{3}$ ice upper limits. This means that the current data set can only be used to search for tentative correlations or to note gross deviations from expected correlations, and not for a proper statistical correlation analysis.

The top lefthand panel of Fig. 10 shows no conclusive correlation between $\mathrm{OCN}^{-}$ice and $\mathrm{HNCO}$ in the gas phase with respect to $\mathrm{CH}_{3} \mathrm{OH}$. Qualitatively, such a correlation is expected since $\mathrm{HNCO}$ and $\mathrm{OCN}^{-}$are linked through efficient thermal acid-base chemistry within the ice (e.g., Demyk et al. 1998; van Broekhuizen et al. 2004; Theule et al. 2011). The lack of a correlation may therefore simply be due to the difficulty determining
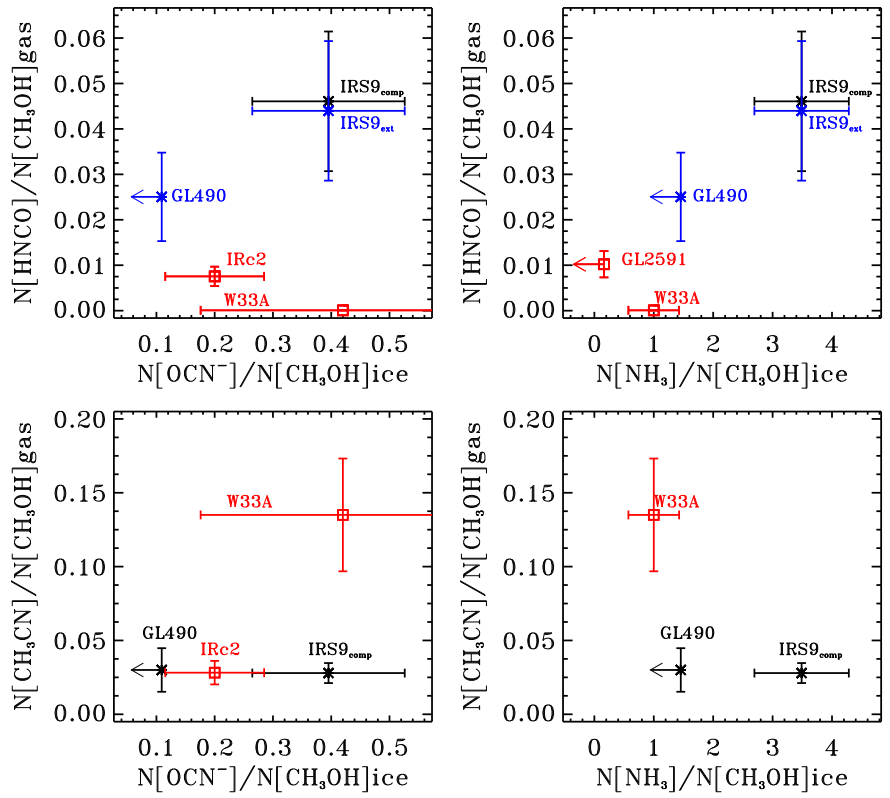

Fig. 10. Ice versus gas abundance correlation for N-bearing species with respect to $\mathrm{CH}_{3} \mathrm{OH}$. The crosses are abundances derived for our organicpoor MYSOs, and the red squares are the values derived by Bisschop et al. (2007). An arbitrary error of $20 \%$ has been assumed for the latter values. For the two top plots, the black crosses represent the HNCO over $\mathrm{CH}_{3} \mathrm{OH}$ abundance derived for the compact component, while the blue crosses correspond to the HNCO abundance calculation for an extended component.

the $\mathrm{OCN}^{-}$abundance in the ice, so we also explore the correlation between $\mathrm{HNCO}$ gas and the better constrained $\mathrm{NH}_{3}$ ice. $\mathrm{NH}_{3}$ is likely the major source of nitrogen in the ice and may therefore be a proxy for the abundance of $\mathrm{N}$-bearing ices in general. It may also affect the $\mathrm{HNCO} / \mathrm{OCN}^{-}$chemistry directly since it is a strong base. The top righthand panel of Fig. 10 shows that there is indeed a tentative correlation between gas phase abundance of $\mathrm{HNCO}$ over $\mathrm{CH}_{3} \mathrm{OH}$ with respect to $\mathrm{NH}_{3}$.

The relation between $\mathrm{OCN}^{-}$in the ice and $\mathrm{CH}_{3} \mathrm{CN}$ in the gas is explored in the bottom lefthand panel of Fig. 10. These molecules do not appear to be correlated for the four sources presented here, despite both containing a $\mathrm{CN}$ functional group. Finally, Rodgers \& Charnley (2001) predict a correlation between $\mathrm{CH}_{3} \mathrm{CN}$ gas a $\mathrm{NH}_{3}$ ice, but in this limited sample we find no correlation between the $\mathrm{CH}_{3} \mathrm{CN} / \mathrm{CH}_{3} \mathrm{OH}$ gas ratio versus the $\mathrm{NH}_{3} / \mathrm{CH}_{3} \mathrm{OH}$ ratio in the ice.

\section{Discussion}

\subsection{Organic-poor MYSOs versus hot cores}

Previous observations of complex molecules toward MYSOs have generally focused on sources with a bright hot core that is responsible for most of the molecular emission. In such cases, either interferometric or single-dish observations are sufficient to determine complex organic abundances as long as the radius of the evaporation front close to the central protostar is known. Single-dish observations combined with the rotational diagram technique can also be used to derive abundances of molecules that are predominantly present in the outer envelope, since beamaveraged abundances can then be assumed. The real difficulty arises for molecules that are distributed throughout the envelope and core. For such molecules, single-dish and interferometric 
observations need to be combined to deduce what fraction of the molecular emission originates in the envelope and in the core, and then use these fractions to calculate the chemical composition of the two physically and chemically different regions. Based on this study, this class of molecules seems to mainly encompass zeroth-generation ices, i.e. $\mathrm{CH}_{3} \mathrm{OH}$ and $\mathrm{HNCO}$, but as our sensitivity increases, we expect, based on model results, that many classical hot core molecules will present a significant envelope emission profile as well (Öberg et al. 2013).

Using the IRAM $30 \mathrm{~m}$ and SMA spectra, we could classify several complex organic molecules as belonging to the core, envelope, and both. The two envelope molecules, $\mathrm{CH}_{3} \mathrm{CHO}$ and $\mathrm{CH}_{3} \mathrm{CCH}$, were similarly classified by Bisschop et al. (2007) based on excitation temperatures alone, suggesting that the envelopes around line-poor MYSOs and hot cores are chemically similar. In contrast we find that in the line-poor MYSOs, $\mathrm{CH}_{3} \mathrm{OH}$ and sometimes HNCO have significant emission contributions from the envelope, while Bisschop et al. (2007) find that in hot core sources, they have excitation temperatures above $100 \mathrm{~K}$ and were thus classified as originating exclusively in the core region; these sources have probably a similar envelope line flux to the one observed for the line-poor MYSOs, but in single-dish studies, this emission contribution is drowned out by the hot cores.

Overall, the chemistry in the young MYSOs is remarkably similar to what is observed in the hot cores, which suggests that they may be hot core precursors. $\mathrm{CH}_{3} \mathrm{CN}, \mathrm{CH}_{3} \mathrm{CCH}, \mathrm{CH}_{3} \mathrm{CHO}$, $\mathrm{HNCO}$, and $\mathrm{CH}_{3} \mathrm{OCH}_{3}$ are observed in both kinds of sources at comparable abundances with respect to $\mathrm{CH}_{3} \mathrm{OH}$. $\mathrm{CH}_{3} \mathrm{CH}_{2} \mathrm{OH}$ and $\mathrm{HCOOCH}_{3}$ - two typical hot-core molecules - are not seen in the organic-poor MYSOs, but typical abundance of these molecules with respect to $\mathrm{CH}_{3} \mathrm{OH}$ from Bisschop et al. (2007) are consistent with non-detections. The hot-core precursor interpretation is also consistent with the observed lack of correlation between $\mathrm{CH}_{3} \mathrm{OH}$ core column density or hot-core activity on the initial $\mathrm{CH}_{3} \mathrm{OH}$ ice abundance.

\subsection{The ice-gas connection: observations vs. theory}

Regardless or whether the overall hot-core chemistry depends on the initial ice composition, we expect that the ice composition will have an effect on the chemical composition in both hot cores and line-poor MYSOs. This dependence may look very different for complex molecules that form in the gas phase from evaporated ices compared to products of complex ice chemistry. That we do not observe a clear trend between $\mathrm{NH}_{3}$ in the ice and $\mathrm{CH}_{3} \mathrm{CN}$ in the gas suggests that the model of Rodgers \& Charnley (2001) is missing important complex molecule formation pathways. We have therefore used the state-of the-art chemical model MAGICKAL (Garrod 2013) to explore the connection between ice and gas phase species further. The model uses a rate-equation/modified rate-equation approach, treating the gas phase, ice surface, and bulk ice as coupled, but distinct, chemical phases.

Garrod (2013) produced generic, single-point hot-core models that treated first a cold collapse to $10^{7} \mathrm{~cm}^{-3}$, followed by a warm-up from 8 to $400 \mathrm{~K}$ at fixed density, assuming a typical grain size of $0.1 \mu \mathrm{m}$ and $10^{6}$ surface binding sites. We reran the warm-up phase, adopting the medium warm-up timescale of $2 \times 10^{5}$ years to reach $200 \mathrm{~K}$ (whose results appear best to fit various other observational results), but altering the ice abundances prior to warm-up. The original $\mathrm{H}_{2} \mathrm{O}$ ice abundance is retained, while the $\mathrm{CH}_{3} \mathrm{OH}, \mathrm{CH}_{4}, \mathrm{HNCO}$, and $\mathrm{NH}_{3}$ values are varied to mimic the observed ranges in the combined line-poor MYSOs and hot cores sample (see M1 to M5 in Table 12). Even though
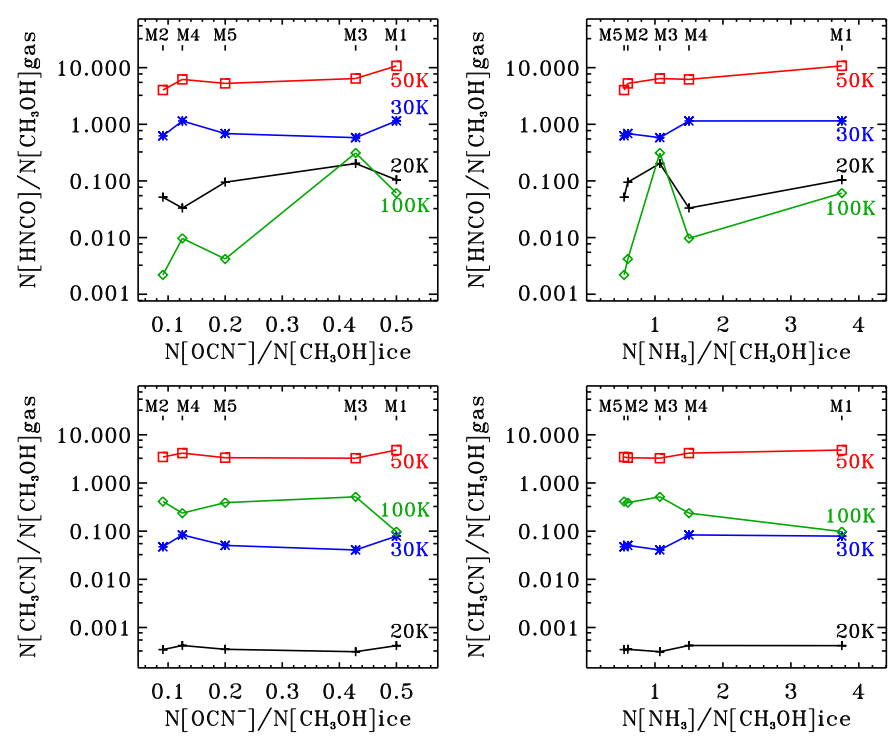

Fig. 11. Model ice versus gas abundance correlation from the MAGICKAL model (Garrod 2013). Five initial ice abundances (M1 to M5) are used to run the models and derived N-bearing abundances with respect to $\mathrm{CH}_{3} \mathrm{OH}$. The black plus signs present the results at $20 \mathrm{~K}$, the blue crosses are the model results at $30 \mathrm{~K}$, the red squares represents $50 \mathrm{~K}$, and the green diamonds are the results at $100 \mathrm{~K}$.

Table 12. Initial ice abundances with respect to water used for the five chemical model simulation M1-5.

\begin{tabular}{llllll}
\hline \hline Species ratio & M1 & M2 & M3 & M4 & M5 \\
\hline $\mathrm{CH}_{3} \mathrm{OH} / \mathrm{H}_{2} \mathrm{O}$ & 4 & 11 & 14 & 4 & 10 \\
$\mathrm{CH}_{4} / \mathrm{H}_{2} \mathrm{O}$ & 2 & 2 & 2 & 2 & 2 \\
$\mathrm{HNCO} / \mathrm{H}_{2} \mathrm{O}$ & 2 & 1 & 6 & 0.5 & 2 \\
$\mathrm{NH}_{3} / \mathrm{H}_{2} \mathrm{O}$ & 15 & 6 & 15 & 6 & 6 \\
\hline
\end{tabular}

Notes. The three-phase model assumes a $\mathrm{H}_{2} \mathrm{O}$ abundance with respect to hydrogen of $2.66 \times 10^{-7} n_{\mathrm{H}}$ for the ice surface and $1.46 \times 10^{-4} n_{\mathrm{H}}$ for the ice bulk.

the ice abundance ratios correspond to the sources in this study, it is not possible to directly compare observations and simulation since key physical parameters such as densities and warm up rates of each specific object is not taken into account. The simulations are instead used here to investigate chemical trends. The resulting gas-phase abundances of $\mathrm{HNCO}, \mathrm{CH}_{3} \mathrm{CN}$, and $\mathrm{CH}_{3} \mathrm{OH}$ are reported at different temperatures during warm-up in Table 13.

The relationships during protostellar warm-up between gasphase $\mathrm{HNCO}$ and $\mathrm{CH}_{3} \mathrm{CN}$ with respect to $\mathrm{CH}_{3} \mathrm{OH}$, and $\mathrm{OCN}^{-}$ and $\mathrm{NH}_{3}$ initial ice abundances with respect to $\mathrm{CH}_{3} \mathrm{OH}$ ice are shown in Fig. 11 for temperatures between $20 \mathrm{~K}$ and $100 \mathrm{~K}$. Since the model does not treat ion chemistry in the ice, a full conversion rate of $\mathrm{HNCO}$ ice into $\mathrm{OCN}^{-}$has been assumed based on the efficient $\mathrm{HNCO}$ to $\mathrm{OCN}^{-}$conversion derived experimentally by Demyk et al. (1998), van Broekhuizen et al. (2004), Theule et al. (2011), among others. The resulting complex molecular gas abundances are regulated by a combination of temperature and initial ice composition. The sensitivity to ice composition varies significantly with temperature however, the gas-phase $\mathrm{HNCO} / \mathrm{CH}_{3} \mathrm{OH}$ ratio, for example, barely changes with ice composition when the grains are sitting at $30 \mathrm{~K}$, because of the very limited sublimation at this temperature. To predict the 
Table 13. Gas abundances with respect to hydrogen at various temperatures derived by the Garrod (2013) model for the initial ice abundances presented in Table 12 .

\begin{tabular}{|c|c|c|c|c|c|}
\hline Model & Species & $n(20 \mathrm{~K}) / n_{\mathrm{H}}$ & $n(30 \mathrm{~K}) / n_{\mathrm{H}}$ & $n(50 \mathrm{~K}) / n_{\mathrm{H}}$ & $n(100 \mathrm{~K}) / n_{\mathrm{H}}$ \\
\hline \multirow[t]{3}{*}{ M1 } & $\mathrm{CH}_{3} \mathrm{OH}$ & $1.6 \times 10^{-11}$ & $1.1 \times 10^{-12}$ & $4.9 \times 10^{-11}$ & $3.2 \times 10^{-9}$ \\
\hline & HNCO & $1.7 \times 10^{-12}$ & $1.2 \times 10^{-12}$ & $5.3 \times 10^{-10}$ & $1.9 \times 10^{-10}$ \\
\hline & $\mathrm{CH}_{3} \mathrm{CN}$ & $6.8 \times 10^{-15}$ & $8.5 \times 10^{-14}$ & $2.4 \times 10^{-10}$ & $3.1 \times 10^{-10}$ \\
\hline \multirow[t]{3}{*}{ M2 } & $\mathrm{CH}_{3} \mathrm{OH}$ & $2.0 \times 10^{-11}$ & $1.9 \times 10^{-12}$ & $1.1 \times 10^{-10}$ & $6.8 \times 10^{-9}$ \\
\hline & HNCO & $1.0 \times 10^{-12}$ & $1.2 \times 10^{-12}$ & $4.5 \times 10^{-10}$ & $1.5 \times 10^{-11}$ \\
\hline & $\mathrm{CH}_{3} \mathrm{CN}$ & $6.8 \times 10^{-15}$ & $8.9 \times 10^{-14}$ & $3.9 \times 10^{-10}$ & $2.8 \times 10^{-9}$ \\
\hline \multirow[t]{3}{*}{ M3 } & $\mathrm{CH}_{3} \mathrm{OH}$ & $2.2 \times 10^{-11}$ & $2.6 \times 10^{-12}$ & $1.1 \times 10^{-10}$ & $7.3 \times 10^{-9}$ \\
\hline & HNCO & $4.4 \times 10^{-12}$ & $1.5 \times 10^{-12}$ & $7.3 \times 10^{-10}$ & $2.3 \times 10^{-9}$ \\
\hline & $\mathrm{CH}_{3} \mathrm{CN}$ & $6.8 \times 10^{-15}$ & $1.1 \times 10^{-13}$ & $3.7 \times 10^{-10}$ & $3.8 \times 10^{-9}$ \\
\hline \multirow[t]{3}{*}{ M4 } & $\mathrm{CH}_{3} \mathrm{OH}$ & $1.6 \times 10^{-11}$ & $9.6 \times 10^{-13}$ & $5.9 \times 10^{-11}$ & $3.7 \times 10^{-9}$ \\
\hline & HNCO & $5.4 \times 10^{-13}$ & $1.1 \times 10^{-12}$ & $3.6 \times 10^{-10}$ & $3.6 \times 10^{-11}$ \\
\hline & $\mathrm{CH}_{3} \mathrm{CN}$ & $6.8 \times 10^{-15}$ & $8.1 \times 10^{-14}$ & $2.4 \times 10^{-10}$ & $8.8 \times 10^{-10}$ \\
\hline \multirow[t]{3}{*}{ M5 } & $\mathrm{CH}_{3} \mathrm{OH}$ & $1.9 \times 10^{-11}$ & $1.8 \times 10^{-12}$ & $9.4 \times 10^{-11}$ & $6.4 \times 10^{-9}$ \\
\hline & HNCO & $1.8 \times 10^{-12}$ & $1.3 \times 10^{-12}$ & $4.9 \times 10^{-10}$ & $2.7 \times 10^{-11}$ \\
\hline & $\mathrm{CH}_{3} \mathrm{CN}$ & $6.8 \times 10^{-15}$ & $9.2 \times 10^{-14}$ & $3.1 \times 10^{-10}$ & $2.5 \times 10^{-10}$ \\
\hline
\end{tabular}

complex chemistry thus clearly requires knowing both the temperature structure and the initial ice composition of a source.

Based on the observational and theoretical results that most $\mathrm{HNCO}$ and $\mathrm{CH}_{3} \mathrm{CN}$ emission start at high temperatures, we focus on the predictions at $100 \mathrm{~K}$. At this temperature, the HNCO gas vs. methanol content is, as expected, correlated to the initial amount of $\mathrm{OCN}^{-}$over methanol in the ice. This result suggests that with more sources and/or better constraints on $\mathrm{OCN}^{-}$ ice abundances, a clearer correlation in the observed data should appear as long as the model captures the dominant HNCO formation/destruction pathways. The observed tentative correlation between gas-phase $\mathrm{HNCO}$ and $\mathrm{NH}_{3}$ ice is consistent with model predictions, except for the M3 run. In the M3 model, the high absolute abundance of HNCO ice results in a longer HNCO desorption time scale, which shifts the abundance peak of HNCO to higher temperatures and results in the high $[\mathrm{HNCO}] /\left[\mathrm{CH}_{3} \mathrm{OH}\right]$ ratio at $100 \mathrm{~K}$; at higher temperatures, M3 no longer deviates from the trend. To fully explore the effects of $\mathrm{NH}_{3}$ and $\mathrm{OCN}^{-}$on the final complex organics abundances clearly requires a much larger grid of models that covers all possible combinations of ice abundances as well as investigating the temperature dependences of the complex chemistry.

In contrast to what has been proposed by Rodgers \& Charnley (2001), the abundance of $\mathrm{CH}_{3} \mathrm{CN}$ with respect to $\mathrm{CH}_{3} \mathrm{OH}$ does not correlate with either the $\mathrm{NH}_{3}$ ice content in the MAGICKAL code output or with the cyanide ice-related species $\mathrm{OCN}^{-}$. This agrees with the observational results. In Rodgers \& Charnley (2001) and Garrod (2013), $\mathrm{CH}_{3} \mathrm{CN}$ forms mainly through radiative association reaction in the gas phase between $\mathrm{CH}_{3}^{+}$and desorbing $\mathrm{HCN}$ giving $\mathrm{CH}_{3} \mathrm{CNH}^{+}$. The correlation between $\mathrm{CH}_{3} \mathrm{CN}$ and $\mathrm{NH}_{3}$ predicted by Rodgers \& Charnley (2001) comes from a cycled production of $\mathrm{HCN}$ from $\mathrm{NH}_{3}$. The latter is, however, not observed in MAGICKAL, which explains the lack of correlation between $\mathrm{CH}_{3} \mathrm{CN}$ and $\mathrm{NH}_{3}$.

In summary, there is some encouraging tentative agreement between model predictions and observations. To directly compare models and observations requires, however, that the appropriate model results are mapped onto the temperature-density profiles of individual sources, since both ice composition and temperature are shown to strongly affect the complex chemistry (Öberg et al. 2013). Thus, to draw any general conclusions requires a large sample of spatially-resolved gas-phase observations, along with ice observations of the same object. As shown here, organic-poor high-mass protostars contain detectable amounts of complex organic material and present a similar chemistry to bright hot cores. Most massive YSOs with existing ice observations could therefore be used to expand the sample of sources.

\section{Conclusions}

We detected complex organic molecules $\mathrm{CH}_{3} \mathrm{CN}, \mathrm{CH}_{3} \mathrm{CCH}$, $\mathrm{CH}_{3} \mathrm{CHO}$, and $\mathrm{CH}_{3} \mathrm{OCH}_{3}$ together with $\mathrm{HNCO}$ and $\mathrm{CH}_{3} \mathrm{OH}$, toward three massive YSOs without any previous evidence of hot-core chemistry activity. Using a combination of singledish and interferometry observations, we found that $\mathrm{CH}_{3} \mathrm{CN}$ and $\mathrm{CH}_{3} \mathrm{OCH}_{3}$ emission originates in the central core region, $\mathrm{CH}_{3} \mathrm{CHO}$ and $\mathrm{CH}_{3} \mathrm{CCH}$ in an extended envelope, and $\mathrm{CH}_{3} \mathrm{OH}$ and, sometimes, HNCO have both envelope and core emission components. The inferred molecular emission locations are consistent with rotational temperatures derived from the single-dish observations, except for $\mathrm{CH}_{3} \mathrm{OH}$, where single-dish data are dominated by the envelope.

The high-temperature abundances of complex organics with respect to $\mathrm{CH}_{3} \mathrm{OH}$ are indistinguishable for the organic-poor MYSOs and the sample of hot core sources from Bisschop et al. (2007) and Isokoski et al. (2013). The envelope chemistry also seems similar for both kinds of sources, but this analysis is limited by a lack of $\mathrm{CH}_{3} \mathrm{OH}$ envelope data toward hot core sources. No strong correlation between initial $\mathrm{CH}_{3} \mathrm{OH}$ ice abundance and hot $\mathrm{CH}_{3} \mathrm{OH}$ gas column density close to the central object was observed.

The $\mathrm{NH}_{3}$ ice abundances seem to affect the $\mathrm{HNCO} / \mathrm{CH}_{3} \mathrm{OH}$ gas-phase abundances. This relationship is reproduced by the MAGICKAL astrochemical code, assuming fiducial collapse and warm-up rates and initial ice compositions that span the observed range. 

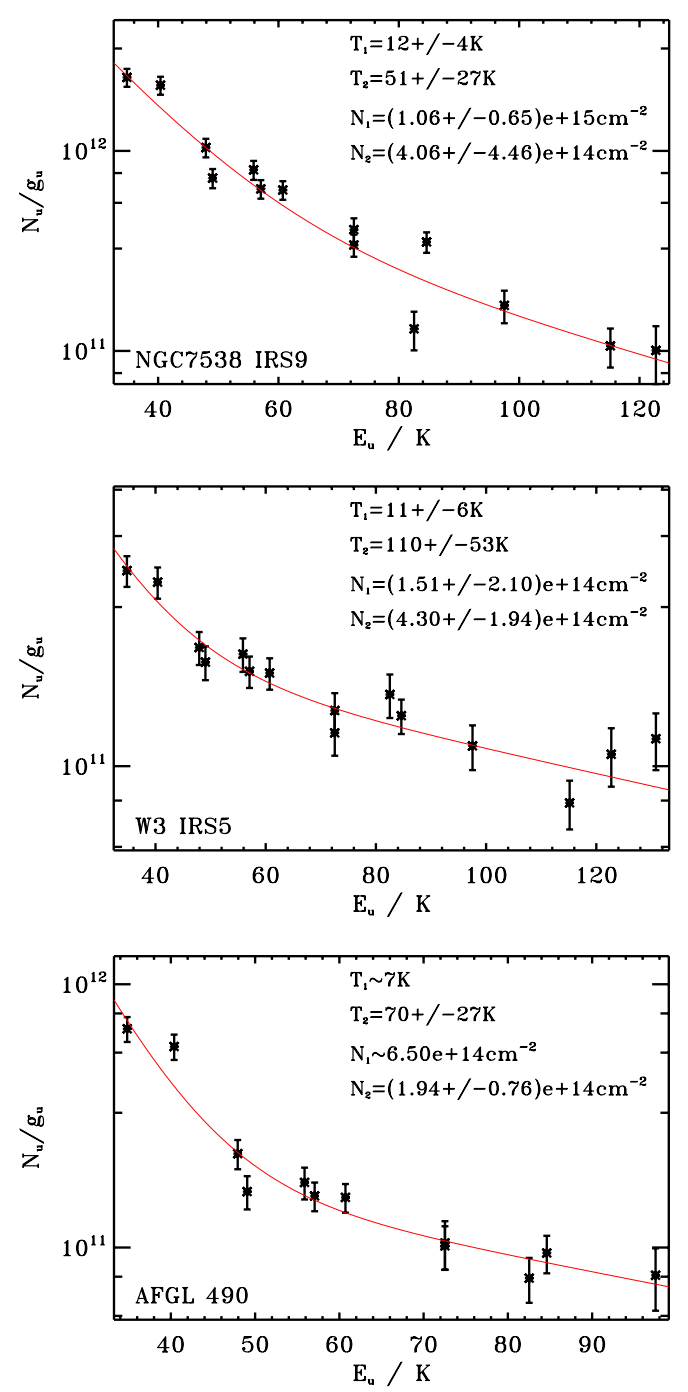

Fig. A.1. Results of a two component fit of the $\mathrm{CH}_{3} \mathrm{OH}$ rotational diagrams for the three line-poor MYSOs using the IRAM $30 \mathrm{~m}$ spectra.

More sources with both ice and gas data are required to settle how ice abundances affect complex molecule distributions around MYSOs. This could be achieved by collecting mid-IR ice spectra (using SOFIA for example), and performing spatially resolved millimetric observations of sources with no detected hotcore molecules.

Acknowledgements. The authors thank the anonymous referee and the editor Malcolm Walmsley for helpful comments and suggestions. E.C.F. is supported by a Rubicon fellowship (680-50-1302), awarded by the Netherlands Organisation for Scientific Research (NWO). R.T.G. is funded by the NASA Astrophysics Theory Program, grant number NNX11AC38G. Astrochemistry in Leiden is supported by the Netherlands Research School for Astronomy (NOVA), by a Royal Netherlands Academy of Arts and Sciences (KNAW) professor prize, and by the European Union A-ERC grant 291141 CHEMPLAN.

\section{Appendix A: Two-component fit of the $\mathrm{CH}_{3} \mathrm{OH}$ rotational diagrams}

The IRAM $30 \mathrm{~m}$ and SMA $\mathrm{CH}_{3} \mathrm{OH}$ line data have been analyzed further to explore whether the data is better fit by two distinct temperature distributions than by the one assumed in the rotational diagrams in the main body text. In fact, two Boltzman distributions would be expected for the IRAM $30 \mathrm{~m}$ data, since these spectra include both the envelope and the warm core seen in the SMA spectra. Figure A.1 presents the two-component rotational diagrams for the three MYSOs. The fits were performed using the IDL routine MPFIT with the initial temperature guesses of $20 \mathrm{~K}$ and $100 \mathrm{~K}$ and initial column densities guesses of $5 \times 10^{14} \mathrm{~cm}^{2}$ and $1 \times 10^{15} \mathrm{~cm}^{2}$.

For all three MYSOs the data is well fit by two components, but the uncertainties in the derived excitation temperatures and column densities are very large, demonstrating that the signalto-noise ratio of this data set is not sufficient for a quantitative two-component analysis. Still, it is clear that in each case, the cold component has a slightly lower excitation temperature than the single-component fit. The derived column densities agrees (taking the large uncertainties into account) between the two fits, supporting our assumption that the IRAM $30 \mathrm{~m} \mathrm{CH}_{3} \mathrm{OH}$ spectra are dominated by the envelope. Each fit also results in a warm component. While the excitation temperatures have large uncertainties, they are all consistent with those derived from the SMA data, further supporting the conclusions based on that data set. Owing to these large uncertainties for the column densities and the hot component temperature, single-temperature fitting was used for the quantitative analysis in the paper. Higher signal-tonoise ratio single-dish data could clearly be used, however, to simultaneously constrain temperature and column densities of the two components, limiting the need for high-spatial-resolution observations for some sources.

We also attempted to fit the $\mathrm{CH}_{3} \mathrm{OH}$ SMA spectra with two temperature components using the same fitting routine and initial guesses. In each case, the outcome was that a single-component fit the data as well as two components; i.e., it was not possible to distinguish multiple components with different temperatures within the SMA masks. This confirms that the SMA beam samples only the hot $\mathrm{CH}_{3} \mathrm{OH}$ component and filters out the extended cold emission.

\section{References}

Bisschop, S. E., Jørgensen, J. K., van Dishoeck, E. F., \& de Wachter, E. B. M. 2007, A\&A, 465, 913

Blake, G. A., Sutton, E. C., \& Masson, C. R. 1987, ApJ, 315, 621

Carter, M., Lazareff, B., Maier, D., et al. 2012, A\&A, 538, A89

Cazaux, S., Tielens, A. G. G. M., Ceccarelli, C., et al. 2003, ApJ, 593, L51

Charnley, S. B., Tielens, A. G. G. M., \& Millar, T. J. 1992, ApJ, 399, L71

Chavarría, L., Herpin, F., Jacq, T., et al. 2010, A\&A, 521, L37

Cuppen, H. M., van Dishoeck, E. F., Herbst, E., \& Tielens, A. G. G. M. 2009, A\&A, 508, 275

Demyk, K., Dartois, E., \& d'Hendecourt, L. 1998, A\&A, 339, 553

Doty, S. D., van Dishoeck, E. F., van der Tak, F. F. S., \& Boonman, A. M. S. 2002, A\&A, 389, 446

Ehrenfreund, P., \& Charnley, S. B. 2000, ARA\&A, 38, 427

Fuchs, G. W., Cuppen, H. M., Ioppolo, S., et al. 2009, A\&A, 505, 629

Fuente, A., Neri, R., \& Caselli, P. 2005, A\&A, 444, 481

Garrod, R. T. 2013, ApJ, 765, 60

Garrod, R. T., Weaver, S. L. W., \& Herbst, E. 2008, ApJ, 682, 283

Gibb, E. L., Whittet, D. C. B., Boogert, A. C., \& Tielens, A. G. G. M. 2004, ApJS, 151, 35

Goldsmith, P. F., \& Langer, W. D. 1999, ApJ, 517, 209

Helmich, F. P., Jansen, D. J., de Graauw, T. H., Groesbeck, T. D., \& van Dishoeck, E. F. 1994, A\&A, 283, 626

Herbst, E., \& van Dishoeck, E. F. 2009, ARA\&A, 47, 427

Isokoski, K., Bottinelli, S., \& van Dishoeck, E. F. 2013, A\&A, 554, A100

Leurini, S., Schilke, P., Wyrowski, F., \& Menten, K. M. 2007, A\&A, 466, 215

Megeath, S. T., Wilson, T. L., \& Corbin, M. R. 2005, ApJ, 622, L141

Mitchell, G. F., Lee, S. W., Maillard, J.-P., et al. 1995, ApJ, 438, 794

Müller, H., Thorwirth, S., Roth, D. A., \& Winnewisser, G. 2001, A\&A, 370, L49

Nomura, H., \& Millar, T. J. 2004, A\&A, 414, 409 
E. C. Fayolle et al.: New constraints on the origins of organics

Öberg, K. I., Garrod, R. T., van Dishoeck, E. F., \& Linnartz, H. 2009, A\&A, 504, 891

Öberg, K. I., Bottinelli, S., Jørgensen, J. K., \& van Dishoeck, E. F. 2010, ApJ, 716,825

Öberg, K. I., Boamah, M. D., Fayolle, E. C., et al. 2013, ApJ, 771, 95

Pickett, H. M., Poynter, R. I., \& Cohen, E. A. 1998, J. Quant. Spectr. Rad. Transf., 60, 883

Rodgers, S. D., \& Charnley, S. B. 2001, ApJ, 546, 324

Rodón, J. A., Beuther, H., Megeath, S. T., \& van der Tak, F. F. S. 2008, A\&A, 490,213

Sandell, G., Goss, W. M., \& Wright, M. 2005, ApJ, 621, 839

Schreyer, K., Semenov, D., Henning, T., \& Forbrich, J. 2006, ApJ, 637, L129

Taban, I. M., Schutte, W. A., Pontoppidan, K. M., \& van Dishoeck, E. F. 2003, A\&A, 399, 169
Theule, P., Duvernay, F. Ilmane, A, et al. 2011, A\&A, 530, A96

Tielens, A. G. G. M., \& Hagen, W. 1982, A\&A, 114, 245

van Broekhuizen, F. A., Keane, J. V., \& Schutte, W. A. 2004, A\&A, 415, 425

van der Tak, F. F. S., van Dishoeck, E. F., \& Caselli, P. 2000, A\&A, 361, 327

van der Tak, F. F. S., Tuthill, P. G., \& Danchi, W. C. 2005, A\&A, 431, 993

Viti, S., Collings, M. P., Dever, J. W., McCoustra, M. R. S., \& Williams, D. A. 2004, MNRAS, 354, 1141

Watanabe, N., \& Kouchi, A. 2002, ApJ, 571, L173

Watanabe, N., Shiraki, T., \& Kouchi, A. 2003, ApJ, 588, L121

Watanabe, N., Nagaoka, A., \& Shiraki, T. 2004, ApJ, 616, 638

Wirström, E. S., Geppert, W. D., Hjalmarson, A., et al. 2011, A\&A, 533, A24

Wyrowski, F., Schilke, P., Walmsley, C. M., \& Menten, K. M. 1999, ApJ, 514, L43 